\title{
Regularized hybrid iterative algorithms for triple hierarchical variational inequalities
}

\author{
Lu-Chuan Ceng ${ }^{1,2}$, Ngai-Ching Wong ${ }^{3,4^{*}}$ and Jen-Chih Yao ${ }^{5,6}$
}

\section{"Correspondence:}

wong@math.nsysu.edu.tw

${ }^{3}$ Department of Applied

Mathematics, National Sun Yat-sen

University, Kaohsiung, 804, Taiwan

${ }^{4}$ Center for General Education,

Kaohsiung Medical University,

Kaohsiung, 807, Taiwan

Full list of author information is

available at the end of the article

\begin{abstract}
In this paper, we introduce and study a triple hierarchical variational inequality (THVI) with constraints of minimization and equilibrium problems. More precisely, let Fix $(T)$ be the fixed point set of a nonexpansive mapping, let $\operatorname{MEP}(\Theta, \varphi)$ be the solution set of a mixed equilibrium problem (MEP), and let $\Gamma$ be the solution set of a minimization problem (MP) for a convex and continuously Frechet differential functional in Hilbert spaces. We want to find a solution $x^{*} \in \operatorname{Fix}(T) \cap \operatorname{MEP}(\Theta, \varphi) \cap \Gamma$ of a variational inequality with a variational inequality constraint over the intersection of Fix $(T), \operatorname{MEP}(\Theta, \varphi)$, and $\Gamma$. We propose a hybrid iterative algorithm with regularization to compute approximate solutions of the THVI, and we present the convergence analysis of the proposed iterative algorithm.

MSC: 49J40; 47J20; 47H10; 65K05; 47H09
\end{abstract}

Keywords: triple hierarchical variational inequality; minimization problem; mixed equilibrium problem; nonexpansive mapping; maximal monotone mapping; regularization

\section{Introduction}

Let $H$ be a Hilbert space with inner product $\langle\cdot, \cdot\rangle$ and norm $\|\cdot\|$ over the real scalar field $\mathbb{R}$. Let $C$ be a nonempty closed convex subset of $H$, and $P_{C}$ be the metric projection of $H$ onto $C$. Let $T: C \rightarrow C$ be a self-mapping on $C$. Denote by $\operatorname{Fix}(T)$ the set of fixed points of $T$. We say that $T$ is L-Lipschitzian if there exists a constant $L \geq 0$ such that

$$
\|T x-T y\| \leq L\|x-y\|, \quad \forall x, y \in C .
$$

When $L=1$ or $0 \leq L<1$, we call $T$ a nonexpansive or a contractive mapping, respectively. We say that a mapping $A: C \rightarrow H$ is $\alpha$-inverse strongly monotone if there exists a constant $\alpha>0$ such that

$$
\langle A x-A y, x-y\rangle \geq \alpha\|A x-A y\|^{2}, \quad \forall x, y \in C,
$$

and that $A$ is $\eta$-strongly monotone (resp. monotone) if there exists a constant $\eta>0$ (resp. $\eta=0)$ such that

$$
\langle A x-A y, x-y\rangle \geq \eta\|x-y\|^{2}, \quad \forall x, y \in C .
$$

@2014 Ceng et al.; licensee Springer. This is an Open Access article distributed under the terms of the Creative Commons Attribution License (http://creativecommons.org/licenses/by/2.0), which permits unrestricted use, distribution, and reproduction in any medium, provided the original work is properly cited. 
It is known that $T$ is nonexpansive if and only if $I-T$ is $\frac{1}{2}$-inverse strongly monotone. Moreover, $L$-Lipschitz continuous mappings are $\frac{1}{L}$-inversely strong monotone (see, e.g., [1]).

Let $f: C \rightarrow \mathbb{R}$ be a convex and continuously Frechet differentiable functional. Consider the minimization problem (MP):

$$
\min _{x \in C} f(x)
$$

(assuming the existence of minimizers). We denote by $\Gamma \neq \emptyset$ the set of minimizers of problem (1.1). The gradient-projection algorithm (GPA) generates a sequence $\left\{x_{n}\right\}$ determined by the gradient $\nabla f$ and the metric projection $P_{C}$ :

$$
x_{n+1}:=P_{C}\left(x_{n}-\lambda \nabla f\left(x_{n}\right)\right), \quad \forall n \geq 0 .
$$

The convergence of algorithm (1.2) depends on $\nabla f$. It is known that if $\nabla f$ is $\eta$-strongly monotone and $L$-Lipschitz continuous, then for $0<\lambda<\frac{2 \eta}{L^{2}}$, the operator

$$
S:=P_{C}(I-\lambda \nabla f)
$$

is a contraction. Hence, the sequence $\left\{x_{n}\right\}$ defined by the GPA (1.2) converges in norm to the unique solution of (1.1). If the gradient $\nabla f$ is only assumed to be Lipschitz continuous, then $\left\{x_{n}\right\}$ can only be weakly convergent when $H$ is infinite-dimensional (a counterexample to the norm convergence of $\left\{x_{n}\right\}$ is given by $\mathrm{Xu}$ [2, Section 5]).

The regularization, in particular the traditional Tikhonov regularization, is usually used to solve ill-posed optimization problems. Consider the regularized minimization problem

$$
\min _{x \in C} f_{\alpha}(x):=f(x)+\frac{\alpha}{2}\|x\|^{2},
$$

where $\alpha>0$ is the regularization parameter, and again $f$ is convex with Lipschitz continuous gradient $\nabla f$. While a regularization method provides the possible strong convergence to the minimum-norm solution, its disadvantage is the implicity. Hence explicit iterative methods seem to be attractive. See, e.g., Xu $[2,3]$.

On the other hand, for a given mapping $A: C \rightarrow H$, we consider the variational inequality problem (VIP) of finding $x^{*} \in C$ such that

$$
\left\langle A x^{*}, x-x^{*}\right\rangle \geq 0, \quad \forall x \in C
$$

The solution set of VIP (1.3) is denoted by $\operatorname{VI}(C, A)$. It is well known that when $A$ is monotone,

$$
x \in \operatorname{VI}(C, A) \quad \Leftrightarrow \quad x=P_{C}(x-\lambda A x), \quad \forall \lambda>0 .
$$

Variational inequality theory has been studied quite extensively and has emerged as an important tool in several branches of pure and applied sciences; see, e.g., $[1,4-8]$ and the references therein. 
When $C$ is the fixed point set $\operatorname{Fix}(T)$ of a nonexpansive mapping $T$ and $A=I-S$, VIP (1.3) becomes the variational inequality problem of finding $x^{*} \in \operatorname{Fix}(T)$ such that

$$
\left\langle(I-S) x^{*}, x-x^{*}\right\rangle \geq 0, \quad \forall x \in \operatorname{Fix}(T)
$$

This problem, introduced by Moudafi and Maingé $[9,10]$, is called the hierarchical fixed point problem. It is clear that if $S$ has fixed points, then they are solutions of VIP (1.4). If $S$ is contractive, the solution set of VIP (1.4) is a singleton and it is well known as a viscosity problem. This was previously introduced by Moudafi [11] and also developed by Xu [12]. In this case, solving VIP (1.4) is equivalent to finding a fixed point of the nonexpansive mapping $P_{\mathrm{Fix}(T)} S$, where $P_{\mathrm{Fix}(T)}$ is the metric projection onto the closed and convex set Fix $(T)$. Yao et al. [8] introduced a two-step algorithm to solve VIP (1.4).

Let $\Theta: C \times C \rightarrow \mathbb{R}$ be a bifunction and $\varphi: C \rightarrow \mathbb{R}$ be a function. Consider the mixed equilibrium problem (MEP) of finding $x \in C$ such that

$$
\Theta(x, y)+\varphi(y)-\varphi(x) \geq 0, \quad \forall y \in C,
$$

which was studied by Ceng and Yao [13]. The solution set of MEP (1.5) is denoted by $\operatorname{MEP}(\Theta, \varphi)$. The MEP (1.5) is very general in the sense that it includes, as special cases, fixed point problems, optimization problems, variational inequality problems, minimax problems, Nash equilibrium problems in noncooperative games and others; see, e.g., [13-15].

Recently, Iiduka $[16,17]$ considered a variational inequality with a variational inequality constraint over the set of fixed points of a nonexpansive mapping. Since this problem has a triple structure in contrast with bilevel programming problems or hierarchical constrained optimization problems or hierarchical fixed point problems, it is referred to as a triple hierarchical constrained optimization problem (THCOP). He presented some examples of THCOP and developed iterative algorithms to find the solution of such a problem. Since the original problem is a variational inequality, in this paper, we call it a triple hierarchical variational inequality (THVI). Ceng et al. introduced and considered some THVI in [18]. A nice survey article on THVI is [19]. See also [20-22].

Extending the works done in [18], we introduce and study in this paper the following triple hierarchical variational inequality with constraints of minimization and equilibrium problems.

\section{The problem to study}

Let $C$ be a nonempty closed convex subset of a real Hilbert space $H$. Let $f: C \rightarrow \mathbb{R}$ be convex and continuously Frechet differentiable with $\Gamma$ being the set of its minimizers. Let $T: C \rightarrow C$ and $S: H \rightarrow H$ be both nonexpansive. Let $V: H \rightarrow H$ be $\rho$-contractive, and $F: C \rightarrow H$ be $\kappa$-Lipschitzian and $\eta$-strongly monotone with constants $\rho \in[0,1)$ and $\kappa, \eta>0$. Suppose $0<\mu<2 \eta / \kappa^{2}$ and $0<\gamma \leq \tau$ where $\tau=1-\sqrt{1-\mu\left(2 \eta-\mu \kappa^{2}\right)}$.

Let $\Xi$ denote the solution set of the following hierarchical variational inequality (HVI): find $z^{*} \in \operatorname{Fix}(T) \cap \operatorname{MEP}(\Theta, \varphi) \cap \Gamma$ such that

$$
\left\langle(\mu F-\gamma S) z^{*}, z-z^{*}\right\rangle \geq 0, \quad \forall z \in \operatorname{Fix}(T) \cap \operatorname{MEP}(\Theta, \varphi) \cap \Gamma,
$$


where the solution set $\Xi$ is assumed to be nonempty. Consider the following triple hierarchical variational inequality (THVI).

Find $x^{*} \in \Xi$ such that

$$
\left\langle(\mu F-\gamma V) x^{*}, x-x^{*}\right\rangle \geq 0, \quad \forall x \in \Xi
$$

Based on the iterative schemes provided by $\mathrm{Xu}$ [2] and the two-step iterative scheme provided by Yao et al. [8], by virtue of the viscosity approximation method, hybrid steepestdescent method and the regularization method, we propose the following hybrid iterative algorithm with regularization:

$$
\begin{cases}\Theta\left(u_{n}, y\right)+\varphi(y)-\varphi\left(u_{n}\right)+\frac{1}{r_{n}}\left\langle y-u_{n}, u_{n}-x_{n}\right\rangle \geq 0, & \forall y \in C, \\ y_{n}=\theta_{n} \gamma S x_{n}+\left(I-\theta_{n} \mu F\right) P_{C}\left(u_{n}-\lambda \nabla f_{\alpha_{n}}\left(u_{n}\right)\right), & \\ x_{n+1}=\beta_{n} \gamma V y_{n}+\left(I-\beta_{n} \mu F\right) T P_{C}\left(u_{n}-\lambda \nabla f_{\alpha_{n}}\left(u_{n}\right)\right), & \forall n \geq 0 .\end{cases}
$$

Here, $0<\lambda<2 / L,\left\{r_{n}\right\},\left\{\alpha_{n}\right\} \subset(0,+\infty)$, and $\left\{\beta_{n}\right\},\left\{\theta_{n}\right\} \subset(0,1)$. It is shown that under appropriate assumptions, the two iterative sequences $\left\{x_{n}\right\}$ and $\left\{y_{n}\right\}$ converge strongly to the unique solution of the THVI (1.6).

\section{Preliminaries}

Let $K$ be a nonempty closed convex subset of a real Hilbert space $H$. We write $x_{n} \rightarrow x$ and $x_{n} \rightarrow x$ to indicate that the sequence $\left\{x_{n}\right\}$ converges weakly and strongly to $x$, respectively. The weak $\omega$-limit set of the sequence $\left\{x_{n}\right\}$ is denoted by

$$
\omega_{w}\left(x_{n}\right):=\left\{x \in H: x_{n_{i}} \rightarrow x \text { for some subsequence }\left\{x_{n_{i}}\right\} \text { of }\left\{x_{n}\right\}\right\} .
$$

The metric (or nearest point) projection from $H$ onto $K$ is the mapping $P_{K}: H \rightarrow K$ which assigns to each point $x \in H$ the unique point $P_{K} x \in K$ satisfying the property

$$
\left\|x-P_{K} x\right\|=\inf _{y \in K}\|x-y\|=: d(x, K) .
$$

Proposition 2.1 For given $x \in H$ and $z \in K$ :

(i) $z=P_{K} x \Leftrightarrow\langle x-z, y-z\rangle \leq 0, \forall y \in K$;

(ii) $z=P_{K} x \Leftrightarrow\|x-z\|^{2} \leq\|x-y\|^{2}-\|y-z\|^{2}, \forall y \in K$;

(iii) $\left\langle P_{K} x-P_{K} y, x-y\right\rangle \geq\left\|P_{K} x-P_{K} y\right\|^{2}, \forall y \in H$.

Hence, $P_{K}$ is nonexpansive and monotone.

Definition 2.2 A mapping $T: H \rightarrow H$ is said to be firmly nonexpansive if $2 T-I$ is nonexpansive, or equivalently,

$$
\langle x-y, T x-T y\rangle \geq\|T x-T y\|^{2}, \quad \forall x, y \in H .
$$

Alternatively, $T$ is firmly nonexpansive if and only if $T$ can be expressed as

$$
T=\frac{1}{2}(I+S),
$$


where $S: H \rightarrow H$ is nonexpansive. Projections are firmly nonexpansive. We call $T$ an $a v$ eraged mapping if $T$ can be expressed as a proper convex combination of the identity map $I$ and a nonexpansive mapping. In particular, firmly nonexpansive mappings are averaged.

Proposition 2.3 (see [23]) Let $T: H \rightarrow H$ be a given mapping.

(i) $T$ is nonexpansive if and only if the complement $I-T$ is $\frac{1}{2}$-inverse strongly monotone.

(ii) If $T$ is $\nu$-inverse strongly monotone, then so is $\gamma T$ for all $\gamma>0$.

(iii) $T$ is averaged if and only if the complement $I-T$ is $v$-inverse strongly monotone for some $v>1 / 2$. Indeed, for $\alpha \in(0,1), T$ is $\alpha$-averaged if and only if $I-T$ is $\frac{1}{2 \alpha}$-inverse strongly monotone.

Proposition 2.4 (see $[23,24])$ Let $S, T, V: H \rightarrow H$.

(i) If $T=(1-\alpha) S+\alpha V$ for some $\alpha \in(0,1)$ and if $S$ is averaged and $V$ is nonexpansive, then $T$ is averaged.

(ii) $T$ is firmly nonexpansive if and only if the complement $I-T$ is firmly nonexpansive.

(iii) If $T=(1-\alpha) S+\alpha V$ for some $\alpha \in(0,1)$ and if $S$ is firmly nonexpansive and $V$ is nonexpansive, then $T$ is averaged.

(iv) The composition of finitely many averaged mappings is averaged. In particular, if $T_{1}$ is $\alpha_{1}$-averaged and $T_{2}$ is $\alpha_{2}$-averaged, where $\alpha_{1}, \alpha_{2} \in(0,1)$, then $T_{1} T_{2}$ is $\left(\alpha_{1}+\alpha_{2}-\alpha_{1} \alpha_{2}\right)$-averaged.

(v) If the mappings $T_{1}, \ldots, T_{N}$ are averaged and have a common fixed point, then

$$
\bigcap_{i=1}^{N} \operatorname{Fix}\left(T_{i}\right)=\operatorname{Fix}\left(T_{1} \cdots T_{N}\right) .
$$

For solving the equilibrium problem for a bifunction $\Theta: C \times C \rightarrow \mathbb{R}$, let us consider the following conditions:

(A1) $\Theta(x, x)=0$ for all $x \in C$;

(A2) $\Theta$ is monotone, that is, $\Theta(x, y)+\Theta(y, x) \leq 0$ for all $x, y \in C$;

(A3) for each $x, y, z \in C, \lim _{t \downarrow 0} \Theta(t z+(1-t) x, y) \leq \Theta(x, y)$;

(A4) for each $x \in C, y \mapsto \Theta(x, y)$ is convex and lower semicontinuous;

(A5) for each $y \in C, x \mapsto \Theta(x, y)$ is weakly upper semicontinuous;

(B1) for each $x \in H$ and $r>0$, there exist a bounded subset $D_{x} \subseteq C$ and $y_{x} \in C$ such that for any $z \in C \backslash D_{x}$,

$$
\Theta\left(z, y_{x}\right)+\varphi\left(y_{x}\right)-\varphi(z)+\frac{1}{r}\left\langle y_{x}-z, z-x\right\rangle<0
$$

(B2) $C$ is a bounded set.

Lemma 2.5 (see [14]) Let $C$ be a nonempty closed convex subset of a real Hilbert space $H$ and $\Theta: C \times C \rightarrow \mathbf{R}$ be a bifunction satisfying (A1)-(A4). Let $r>0$ and $x \in H$. Then there exists $z \in C$ such that

$$
\Theta(z, y)+\frac{1}{r}\langle y-z, z-x\rangle \geq 0, \quad \forall y \in C .
$$


Lemma 2.6 (see [15]) Let $C$ be a nonempty closed convex subset of a real Hilbert space $H$. Let $\Theta: C \times C \rightarrow \mathbb{R}$ be a bifunction satisfying (A1)-(A5) and $\varphi: C \rightarrow \mathbb{R}$ be a proper lower semicontinuous and convex function. For $r>0$ and $x \in H$, define a mapping $T_{r}: H \rightarrow C$ as follows:

$$
T_{r} x:=\left\{z \in C: \Theta(z, y)+\varphi(y)-\varphi(z)+\frac{1}{r}\langle y-z, z-x\rangle \geq 0, \forall y \in C\right\}
$$

for all $x \in H$. Assume that either (B1) or (B2) holds. Then $T_{r}$ is a single-valued firmly nonexpansive map on $H$, and $\operatorname{Fix}\left(T_{r}\right)=\operatorname{MEP}(\Theta, \varphi)$ is closed and convex.

Lemma 2.7 (see [25]) Let $\left\{a_{n}\right\}$ be a sequence of nonnegative real numbers such that

$$
a_{n+1} \leq\left(1-s_{n}\right) a_{n}+s_{n} t_{n}+\epsilon_{n}, \quad \forall n \geq 0 .
$$

Here, $0<s_{n} \leq 1,0 \leq \epsilon_{n}$, and $t_{n} \in \mathbb{R}$ for all $n=0,1,2, \ldots$, such that

(i) $\sum_{n=0}^{\infty} s_{n}=+\infty$;

(ii) either $\limsup _{n \rightarrow \infty} t_{n} \leq 0$ or $\sum_{n=0}^{\infty} s_{n}\left|t_{n}\right|<+\infty$;

(iii) $\sum_{n=0}^{\infty} \epsilon_{n}<+\infty$.

Then $\lim _{n \rightarrow \infty} a_{n}=0$.

Lemma 2.8 (Demiclosedness principle; see [1]) Let C be a nonempty closed convex subset of a real Hilbert space $H$ and let $T: C \rightarrow C$ be a nonexpansive mapping with $\operatorname{Fix}(T) \neq \emptyset$. If $\left\{x_{n}\right\}$ is a sequence in $C$ converging weakly to $x$ and if $\left\{(I-T) x_{n}\right\}$ converges strongly to $y$, then $(I-T) x=y$; in particular, if $y=0$, then $x \in \operatorname{Fix}(T)$.

Lemma 2.9 (see [12]) Let $S: H \rightarrow H$ be a nonexpansive mapping and $V: H \rightarrow H$ be a $\rho$-contraction with $\rho \in[0,1)$, respectively.

(i) $I-S$ is monotone, i.e.,

$$
\langle(I-S) x-(I-S) y, x-y\rangle \geq 0, \quad \forall x, y \in H .
$$

(ii) $I-V$ is $(1-\rho)$-strongly monotone, i.e.,

$$
\langle(I-V) x-(I-V) y, x-y\rangle \geq(1-\rho)\|x-y\|^{2}, \quad \forall x, y \in H .
$$

Lemma 2.10 ([26]) Let $H$ be a real Hilbert space. Then, for all $x, y \in H$ and $\lambda \in[0,1]$,

$$
\|\lambda x+(1-\lambda) y\|^{2}=\lambda\|x\|^{2}+(1-\lambda)\|y\|^{2}-\lambda(1-\lambda)\|x-y\|^{2} .
$$

Lemma 2.11 We have the following inequality in an inner product space X:

$$
\|x+y\|^{2} \leq\|x\|^{2}+2\langle y, x+y\rangle, \quad \forall x, y \in X .
$$

Notations Let $\lambda$ be a number in $(0,1]$ and let $\mu, \kappa, \eta>0$. Let $F: C \rightarrow H$ be $\kappa$-Lipschitzian and $\eta$-strongly monotone. Associated with a nonexpansive mapping $T: C \rightarrow C$, we define the mapping $T^{\lambda}: C \rightarrow H$ by

$$
T^{\lambda} x:=(I-\lambda \mu F) T x, \quad \forall x \in C .
$$


Lemma 2.12 (see [27, Lemma 3.1]) The map $T^{\lambda}$ is a contraction provided $\mu<2 \eta / \kappa^{2}$, that is,

$$
\left\|T^{\lambda} x-T^{\lambda} y\right\| \leq(1-\lambda \tau)\|x-y\|, \quad \forall x, y \in C,
$$

where $\tau=1-\sqrt{1-\mu\left(2 \eta-\mu \kappa^{2}\right)} \in(0,1]$. In particular, if $T=I$ the identity mapping, then

$$
\|(I-\lambda \mu F) x-(I-\lambda \mu F) y\| \leq(1-\lambda \tau)\|x-y\|, \quad \forall x, y \in C .
$$

A set-valued mapping $\widetilde{T}: H \rightarrow 2^{H}$ is called monotone if $\langle x-y, f-g\rangle \geq 0$ for all $x, y \in H$, $f \in \widetilde{T} x$ and $g \in \widetilde{T} y$. A monotone set-valued mapping $\widetilde{T}: H \rightarrow 2^{H}$ is called maximal if its graph $\operatorname{Gph}(\widetilde{T})$ is not properly contained in the graph of any other monotone set-valued mapping. It is known that a monotone set-valued mapping $\widetilde{T}: H \rightarrow 2^{H}$ is maximal if and only if for $(x, f) \in H \times H,\langle x-y, f-g\rangle \geq 0$ for every $(y, g) \in \operatorname{Gph}(\widetilde{T})$ implies that $f \in \widetilde{T} x$.

Let $A: C \rightarrow H$ be a monotone and Lipschitz continuous mapping and let $N_{C} v$ be the normal cone to $C$ at $v \in C$, namely

$$
N_{C} v=\{w \in H:\langle v-u, w\rangle \geq 0, \forall u \in C\} .
$$

Define

$$
\widetilde{T} v= \begin{cases}A v+N_{C} v, & \text { if } v \in C \\ \emptyset, & \text { if } v \notin C .\end{cases}
$$

Lemma 2.13 (see [28]) Let $A: C \rightarrow H$ be a monotone mapping.

(i) $\widetilde{T}$ is maximal monotone;

(ii) $v \in \widetilde{T}^{-1} 0 \Leftrightarrow v \in \mathrm{VI}(C, A)$.

\section{Main results}

Let us consider the following three-step iterative scheme with regularization:

$$
\left\{\begin{array}{l}
\Theta\left(u_{n}, y\right)+\varphi(y)-\varphi\left(u_{n}\right)+\frac{1}{r_{n}}\left\langle y-u_{n}, u_{n}-x_{n}\right\rangle \geq 0, \quad \forall y \in C, \\
y_{n}=\theta_{n} \gamma S x_{n}+\left(I-\theta_{n} \mu F\right) P_{C}\left(u_{n}-\lambda \nabla f_{\alpha_{n}}\left(u_{n}\right)\right), \\
x_{n+1}=\beta_{n} \gamma V y_{n}+\left(I-\beta_{n} \mu F\right) T P_{C}\left(u_{n}-\lambda \nabla f_{\alpha_{n}}\left(u_{n}\right)\right), \quad \forall n=0,1,2, \ldots
\end{array}\right.
$$

Here,

- $V: H \rightarrow H$ is a $\rho$-contraction;

- $S: H \rightarrow H$ and $T: C \rightarrow C$ are nonexpansive mappings;

- $F: C \rightarrow H$ is a $\kappa$-Lipschitzian and $\eta$-strongly monotone mapping;

- $\Theta: C \times C \rightarrow \mathbb{R}$ and $\varphi: C \rightarrow \mathbb{R}$ are real-valued functions;

- $\nabla f: C \rightarrow H$ is $L$-Lipschitz continuous with $0<\lambda<\frac{2}{L}$;

- $\left\{r_{n}\right\}$ and $\left\{\alpha_{n}\right\}$ are sequences in $(0,+\infty)$ with $\sum_{n=0}^{\infty} \alpha_{n}<+\infty$ and $\liminf _{n \rightarrow \infty} r_{n}>0$;

- $\left\{\beta_{n}\right\}$ and $\left\{\theta_{n}\right\}$ are sequences in $(0,1)$;

- $0<\mu<2 \eta / \kappa^{2}$ and $0<\gamma \leq \tau$, where $\tau=1-\sqrt{1-\mu\left(2 \eta-\mu \kappa^{2}\right)}$.

Theorem 3.1 Suppose that $\Theta: C \times C \rightarrow \mathbb{R}$ satisfies (A1)-(A5) and that (B1) or (B2) holds. Let $\left\{x_{n}\right\}$ be the bounded sequence generated from any given $x_{0} \in C$ by (3.1). Assume that 
(H1) $\sum_{n=0}^{\infty} \beta_{n}=+\infty, \lim _{n \rightarrow \infty} \frac{1}{\beta_{n}}\left|1-\frac{\theta_{n-1}}{\theta_{n}}\right|=0$;

(H2) $\lim _{n \rightarrow \infty} \frac{1}{\beta_{n}}\left|\frac{1}{\theta_{n}}-\frac{1}{\theta_{n-1}}\right|=0$ and $\lim _{n \rightarrow \infty} \frac{1}{\theta_{n}}\left|1-\frac{\beta_{n-1}}{\beta_{n}}\right|=0$;

(H3) $\lim _{n \rightarrow \infty} \theta_{n}=0, \lim _{n \rightarrow \infty} \frac{\alpha_{n}+\beta_{n}}{\theta_{n}}=0$, and $\lim _{n \rightarrow \infty} \frac{\theta_{n}^{2}}{\beta_{n}}=0$;

(H4) $\lim _{n \rightarrow \infty} \frac{\left|\alpha_{n}-\alpha_{n-1}\right|}{\beta_{n} \theta_{n}}=0$ and $\lim _{n \rightarrow \infty} \frac{\left|r_{n}-r_{n-1}\right|}{\beta_{n} \theta_{n}}=0$.

Then we have the following:

(i) $\lim _{n \rightarrow \infty} \frac{\left\|x_{n+1}-x_{n}\right\|}{\theta_{n}}=0$;

(ii) $\omega_{w}\left(x_{n}\right) \subset \operatorname{Fix}(T) \cap \operatorname{MEP}(\Theta, \varphi) \cap \Gamma$;

(iii) $\omega_{w}\left(x_{n}\right) \subset \Xi$ if $\left\|x_{n}-y_{n}\right\|=o\left(\theta_{n}\right)$ held in addition, i.e., $\lim _{n \rightarrow \infty} \frac{\left\|x_{n}-y_{n}\right\|}{\theta_{n}}=0$.

Proof First, let us show that $P_{C}\left(I-\lambda \nabla f_{\alpha}\right)$ is $\xi$-averaged for each $\lambda \in\left(0, \frac{2}{\alpha+L}\right)$, where

$$
\xi=\frac{2+\lambda(\alpha+L)}{4} \in(0,1)
$$

The Lipschitz condition implies that the gradient $\nabla f$ is $\frac{1}{L}$-inverse strongly monotone [1], that is,

$$
\langle\nabla f(x)-\nabla f(y), x-y\rangle \geq \frac{1}{L}\|\nabla f(x)-\nabla f(y)\|^{2}
$$

Observe that

$$
\begin{aligned}
(\alpha & +L)\left\langle\nabla f_{\alpha}(x)-\nabla f_{\alpha}(y), x-y\right\rangle \\
& =(\alpha+L)\left[\alpha\|x-y\|^{2}+\langle\nabla f(x)-\nabla f(y), x-y\rangle\right] \\
& =\alpha^{2}\|x-y\|^{2}+\alpha\langle\nabla f(x)-\nabla f(y), x-y\rangle+\alpha L\|x-y\|^{2}+L\langle\nabla f(x)-\nabla f(y), x-y\rangle \\
& \geq \alpha^{2}\|x-y\|^{2}+2 \alpha\langle\nabla f(x)-\nabla f(y), x-y\rangle+\|\nabla f(x)-\nabla f(y)\|^{2} \\
& =\|\alpha(x-y)+\nabla f(x)-\nabla f(y)\|^{2} \\
& =\left\|\nabla f_{\alpha}(x)-\nabla f_{\alpha}(y)\right\|^{2} .
\end{aligned}
$$

Hence, $\nabla f_{\alpha}=\alpha I+\nabla f$ is $\frac{1}{\alpha+L}$-inverse strongly monotone. Thus, $\lambda \nabla f_{\alpha}$ is $\frac{1}{\lambda(\alpha+L)}$-inverse strongly monotone by Proposition 2.3(ii). By Proposition 2.3(iii) the complement $I-\lambda \nabla f_{\alpha}$ is $\frac{\lambda(\alpha+L)}{2}$-averaged. Noting that $P_{C}$ is $\frac{1}{2}$-averaged and utilizing Proposition 2.4(iv), we know that for each $\lambda \in\left(0, \frac{2}{\alpha+L}\right)$, the map $P_{C}\left(I-\lambda \nabla f_{\alpha}\right)$ is $\xi$-averaged with

$$
\xi=\frac{1}{2}+\frac{\lambda(\alpha+L)}{2}-\frac{1}{2} \cdot \frac{\lambda(\alpha+L)}{2}=\frac{2+\lambda(\alpha+L)}{4} \in(0,1) .
$$

In particular, $P_{C}\left(I-\lambda \nabla f_{\alpha}\right)$ is nonexpansive. Furthermore, for $\lambda \in\left(0, \frac{2}{L}\right)$, utilizing the fact that $\lim _{n \rightarrow \infty} \frac{2}{\alpha_{n}+L}=\frac{2}{L}$, we may assume

$$
0<\lambda<\frac{2}{\alpha_{n}+L}, \quad \forall n \geq 0 .
$$

Consequently, for each integer $n \geq 0, P_{C}\left(I-\lambda \nabla f_{\alpha_{n}}\right)$ is $\xi_{n}$-averaged with

$$
\xi_{n}=\frac{1}{2}+\frac{\lambda\left(\alpha_{n}+L\right)}{2}-\frac{1}{2} \cdot \frac{\lambda\left(\alpha_{n}+L\right)}{2}=\frac{2+\lambda\left(\alpha_{n}+L\right)}{4} \in(0,1) .
$$

This immediately implies that $P_{C}\left(I-\lambda \nabla f_{\alpha_{n}}\right)$ is nonexpansive for all $n \geq 0$. 
We divide the proof into several steps.

Step 1. $\lim _{n \rightarrow \infty} \frac{\left\|x_{n+1}-x_{n}\right\|}{\theta_{n}}=0$.

For simplicity, put $\tilde{u}_{n}=P_{C}\left(u_{n}-\lambda \nabla f_{\alpha_{n}}\left(u_{n}\right)\right)$. Then $y_{n}=\theta_{n} \gamma S x_{n}+\left(I-\theta_{n} \mu F\right) \tilde{u}_{n}$ and $x_{n+1}=$ $\beta_{n} \gamma V y_{n}+\left(I-\beta_{n} \mu F\right) T \tilde{u}_{n}$ for every $n \geq 0$. We observe that

$$
\begin{aligned}
\left\|\tilde{u}_{n}-\tilde{u}_{n-1}\right\| \leq & \left\|P_{C}\left(I-\lambda \nabla f_{\alpha_{n}}\right) u_{n}-P_{C}\left(I-\lambda \nabla f_{\alpha_{n}}\right) u_{n-1}\right\| \\
& +\left\|P_{C}\left(I-\lambda \nabla f_{\alpha_{n}}\right) u_{n-1}-P_{C}\left(I-\lambda \nabla f_{\alpha_{n-1}}\right) u_{n-1}\right\| \\
\leq & \left\|u_{n}-u_{n-1}\right\|+\left\|P_{C}\left(I-\lambda \nabla f_{\alpha_{n}}\right) u_{n-1}-P_{C}\left(I-\lambda \nabla f_{\alpha_{n-1}}\right) u_{n-1}\right\| \\
\leq & \left\|u_{n}-u_{n-1}\right\|+\left\|\left(I-\lambda \nabla f_{\alpha_{n}}\right) u_{n-1}-\left(I-\lambda \nabla f_{\alpha_{n-1}}\right) u_{n-1}\right\| \\
= & \left\|u_{n}-u_{n-1}\right\|+\left\|\lambda \nabla f_{\alpha_{n}}\left(u_{n-1}\right)-\lambda \nabla f_{\alpha_{n-1}}\left(u_{n-1}\right)\right\| \\
= & \left\|u_{n}-u_{n-1}\right\|+\lambda\left|\alpha_{n}-\alpha_{n-1}\right|\left\|u_{n-1}\right\|, \quad \forall n \geq 1 .
\end{aligned}
$$

Moreover, from (3.1) we have

$$
\left\{\begin{array}{l}
y_{n}=\theta_{n} \gamma S x_{n}+\left(I-\theta_{n} \mu F\right) \tilde{u}_{n}, \\
y_{n-1}=\theta_{n-1} \gamma S x_{n-1}+\left(I-\theta_{n-1} \mu F\right) \tilde{u}_{n-1}, \quad \forall n \geq 1 .
\end{array}\right.
$$

Thus

$$
\begin{aligned}
y_{n}-y_{n-1}= & \theta_{n}\left(\gamma S x_{n}-\gamma S x_{n-1}\right)+\left(\theta_{n}-\theta_{n-1}\right)\left(\gamma S x_{n-1}-\mu F \tilde{u}_{n-1}\right) \\
& +\left(I-\theta_{n} \mu F\right) \tilde{u}_{n}-\left(I-\theta_{n} \mu F\right) \tilde{u}_{n-1} .
\end{aligned}
$$

Utilizing Lemma 2.12 from (3.2) we deduce that

$$
\begin{aligned}
\left\|y_{n}-y_{n-1}\right\| \leq & \theta_{n}\left\|\gamma S x_{n}-\gamma S x_{n-1}\right\|+\left|\theta_{n}-\theta_{n-1}\right|\left\|\gamma S x_{n-1}-\mu F \tilde{u}_{n-1}\right\| \\
& +\left\|\left(I-\theta_{n} \mu F\right) \tilde{u}_{n}-\left(I-\theta_{n} \mu F\right) \tilde{u}_{n-1}\right\| \\
\leq & \theta_{n} \gamma\left\|x_{n}-x_{n-1}\right\|+\left|\theta_{n}-\theta_{n-1}\right|\left\|\gamma S x_{n-1}-\mu F \tilde{u}_{n-1}\right\| \\
& +\left(1-\theta_{n} \tau\right)\left\|\tilde{u}_{n}-\tilde{u}_{n-1}\right\| \\
\leq & \theta_{n} \gamma\left\|x_{n}-x_{n-1}\right\|+\left|\theta_{n}-\theta_{n-1}\right|\left\|\gamma S x_{n-1}-\mu F \tilde{u}_{n-1}\right\| \\
& +\left(1-\theta_{n} \tau\right)\left(\left\|u_{n}-u_{n-1}\right\|+\lambda\left|\alpha_{n}-\alpha_{n-1}\right|\left\|u_{n-1}\right\|\right)
\end{aligned}
$$

where $\tau=1-\sqrt{1-\mu\left(2 \eta-\mu \kappa^{2}\right)}$. Taking into consideration that $u_{n}=T_{r_{n}} x_{n}$ and $u_{n-1}=$ $T_{r_{n-1}} x_{n-1}$, we have

$$
\Theta\left(u_{n}, y\right)+\varphi(y)-\varphi\left(u_{n}\right)+\frac{1}{r_{n}}\left\langle y-u_{n}, u_{n}-x_{n}\right\rangle \geq 0, \quad \forall y \in C
$$

and

$$
\Theta\left(u_{n-1}, y\right)+\varphi(y)-\varphi\left(u_{n-1}\right)+\frac{1}{r_{n-1}}\left\langle y-u_{n-1}, u_{n-1}-x_{n-1}\right\rangle \geq 0, \quad \forall y \in C .
$$

Putting $y=u_{n-1}$ in (3.4) and $y=u_{n}$ in (3.5), we obtain

$$
\Theta\left(u_{n}, u_{n-1}\right)+\varphi\left(u_{n-1}\right)-\varphi\left(u_{n}\right)+\frac{1}{r_{n}}\left\langle u_{n-1}-u_{n}, u_{n}-x_{n}\right\rangle \geq 0, \quad \forall y \in C
$$


and

$$
\Theta\left(u_{n-1}, u_{n}\right)+\varphi\left(u_{n}\right)-\varphi\left(u_{n-1}\right)+\frac{1}{r_{n-1}}\left\langle u_{n}-u_{n-1}, u_{n-1}-x_{n-1}\right\rangle \geq 0, \quad \forall y \in C .
$$

Adding the last two inequalities, by (A2) we get

$$
\left\langle u_{n}-u_{n-1}, \frac{u_{n-1}-x_{n-1}}{r_{n-1}}-\frac{u_{n}-x_{n}}{r_{n}}\right\rangle \geq 0,
$$

and hence

$$
\left\langle u_{n}-u_{n-1}, u_{n-1}-u_{n}+u_{n}-x_{n-1}-\frac{r_{n-1}}{r_{n}}\left(u_{n}-x_{n}\right)\right\rangle \geq 0 .
$$

Since $\liminf _{n \rightarrow \infty} r_{n}>0$, we may assume, without loss of generality, that there exists a positive number $c$ such that $r_{n} \geq c>0$ for all $n \geq 0$. Thus we have

$$
\begin{aligned}
\left\|u_{n}-u_{n-1}\right\|^{2} & \leq\left\langle u_{n}-u_{n-1}, x_{n}-x_{n-1}+\left(1-\frac{r_{n-1}}{r_{n}}\right)\left(u_{n}-x_{n}\right)\right\rangle \\
& \leq\left\|u_{n}-u_{n-1}\right\|\left[\left\|x_{n}-x_{n-1}\right\|+\left|1-\frac{r_{n-1}}{r_{n}}\right|\left\|u_{n}-x_{n}\right\|\right],
\end{aligned}
$$

and hence

$$
\begin{aligned}
\left\|u_{n}-u_{n-1}\right\| & \leq\left\|x_{n}-x_{n-1}\right\|+\left|1-\frac{r_{n-1}}{r_{n}}\right|\left\|u_{n}-x_{n}\right\| \\
& \leq\left\|x_{n}-x_{n-1}\right\|+\frac{M_{0}}{c}\left|r_{n}-r_{n-1}\right| .
\end{aligned}
$$

Here, $M_{0}=\sup \left\{\left\|u_{n}-x_{n}\right\|: n \geq 0\right\}<+\infty$.

Substituting (3.6) into (3.3) we derive

$$
\begin{aligned}
\left\|y_{n}-y_{n-1}\right\| \leq & \theta_{n} \gamma\left\|x_{n}-x_{n-1}\right\|+\left|\theta_{n}-\theta_{n-1}\right|\left\|\gamma S x_{n-1}-\mu F \tilde{u}_{n-1}\right\| \\
& +\left(1-\theta_{n} \tau\right)\left(\left\|x_{n}-x_{n-1}\right\|+\frac{M_{0}}{c}\left|r_{n}-r_{n-1}\right|+\lambda\left|\alpha_{n}-\alpha_{n-1}\right|\left\|u_{n-1}\right\|\right) \\
\leq & \left(1-\theta_{n}(\tau-\gamma)\right)\left\|x_{n}-x_{n-1}\right\|+\left|\theta_{n}-\theta_{n-1}\right|\left\|\gamma S x_{n-1}-\mu F \tilde{u}_{n-1}\right\| \\
& +\frac{M_{0}}{c}\left|r_{n}-r_{n-1}\right|+\lambda\left|\alpha_{n}-\alpha_{n-1}\right|\left\|u_{n-1}\right\| \\
\leq & \left\|x_{n}-x_{n-1}\right\|+M_{1}\left(\left|\theta_{n}-\theta_{n-1}\right|+\left|r_{n}-r_{n-1}\right|+\left|\alpha_{n}-\alpha_{n-1}\right|\right) .
\end{aligned}
$$

Here, $\left\|\gamma S x_{n}-\mu F \tilde{u}_{n}\right\|+\frac{M_{0}}{c}+\lambda\left\|u_{n}\right\| \leq M_{1}$ for some $M_{1} \geq 0$.

On the other hand, from (3.1) we have

$$
\left\{\begin{array}{l}
x_{n+1}=\beta_{n} \gamma V y_{n}+\left(I-\beta_{n} \mu F\right) T \tilde{u}_{n}, \\
x_{n}=\beta_{n-1} \gamma V y_{n-1}+\left(I-\beta_{n-1} \mu F\right) T \tilde{u}_{n-1}, \quad \forall n \geq 1 .
\end{array}\right.
$$

Simple calculations show that

$$
\begin{aligned}
x_{n+1}-x_{n}= & \left(I-\beta_{n} \mu F\right) T \tilde{u}_{n}-\left(I-\beta_{n} \mu F\right) T \tilde{u}_{n-1} \\
& +\left(\beta_{n}-\beta_{n-1}\right)\left(\gamma V y_{n-1}-\mu F T \tilde{u}_{n-1}\right)+\beta_{n}\left(\gamma V y_{n}-\gamma V y_{n-1}\right) .
\end{aligned}
$$


Utilizing Lemma 2.12 from (3.2), (3.6), and (3.7) we deduce that

$$
\begin{aligned}
& \left\|x_{n+1}-x_{n}\right\| \\
& \leq\left\|\left(I-\beta_{n} \mu F\right) T \tilde{u}_{n}-\left(I-\beta_{n} \mu F\right) T \tilde{u}_{n-1}\right\|+\left|\beta_{n}-\beta_{n-1}\right|\left\|\gamma V y_{n-1}-\mu F T \tilde{u}_{n-1}\right\| \\
& +\beta_{n}\left\|\gamma V y_{n}-\gamma V y_{n-1}\right\| \\
& \leq\left(1-\beta_{n} \tau\right)\left\|\tilde{u}_{n}-\tilde{u}_{n-1}\right\|+\left|\beta_{n}-\beta_{n-1}\right|\left\|\gamma V y_{n-1}-\mu F T \tilde{u}_{n-1}\right\|+\beta_{n} \gamma \rho\left\|y_{n}-y_{n-1}\right\| \\
& \leq\left(1-\beta_{n} \tau\right)\left(\left\|u_{n}-u_{n-1}\right\|+\lambda\left|\alpha_{n}-\alpha_{n-1}\right|\left\|u_{n-1}\right\|\right)+\left|\beta_{n}-\beta_{n-1}\right|\left\|\gamma V y_{n-1}-\mu F T \tilde{u}_{n-1}\right\| \\
& +\beta_{n} \gamma \rho\left\|y_{n}-y_{n-1}\right\| \\
& \leq\left(1-\beta_{n} \tau\right)\left(\left\|x_{n}-x_{n-1}\right\|+\frac{M_{0}}{c}\left|r_{n}-r_{n-1}\right|+\lambda\left|\alpha_{n}-\alpha_{n-1}\right|\left\|u_{n-1}\right\|\right) \\
& +\left|\beta_{n}-\beta_{n-1}\right|\left\|\gamma V y_{n-1}-\mu F T \tilde{u}_{n-1}\right\|+\beta_{n} \gamma \rho\left[\left\|x_{n}-x_{n-1}\right\|+M_{1}\left(\left|\theta_{n}-\theta_{n-1}\right|\right.\right. \\
& \left.\left.+\left|r_{n}-r_{n-1}\right|+\left|\alpha_{n}-\alpha_{n-1}\right|\right)\right] \\
& \leq\left(1-\beta_{n} \tau\right)\left[\left\|x_{n}-x_{n-1}\right\|+M_{1}\left(\left|r_{n}-r_{n-1}\right|+\left|\alpha_{n}-\alpha_{n-1}\right|\right)\right] \\
& +\left|\beta_{n}-\beta_{n-1}\right|\left\|\gamma V y_{n-1}-\mu F T \tilde{u}_{n-1}\right\| \\
& +\beta_{n} \gamma \rho\left[\left\|x_{n}-x_{n-1}\right\|+M_{1}\left(\left|\theta_{n}-\theta_{n-1}\right|+\left|r_{n}-r_{n-1}\right|+\left|\alpha_{n}-\alpha_{n-1}\right|\right)\right] \\
& \leq\left(1-\beta_{n} \tau\right)\left\|x_{n}-x_{n-1}\right\|+\left(1-\beta_{n} \tau\right) M_{1}\left(\left|r_{n}-r_{n-1}\right|+\left|\alpha_{n}-\alpha_{n-1}\right|\right) \\
& +\left|\beta_{n}-\beta_{n-1}\right|\left\|\gamma V y_{n-1}-\mu F T \tilde{u}_{n-1}\right\| \\
& +\beta_{n} \gamma \rho\left\|x_{n}-x_{n-1}\right\|+\beta_{n} \tau M_{1}\left(\left|\theta_{n}-\theta_{n-1}\right|+\left|r_{n}-r_{n-1}\right|+\left|\alpha_{n}-\alpha_{n-1}\right|\right) \\
& \leq\left(1-\beta_{n}(\tau-\gamma \rho)\right)\left\|x_{n}-x_{n-1}\right\|+\left|\beta_{n}-\beta_{n-1}\right|\left\|\gamma V y_{n-1}-\mu F T \tilde{u}_{n-1}\right\| \\
& +M_{1}\left(\left|\theta_{n}-\theta_{n-1}\right|+\left|r_{n}-r_{n-1}\right|+\left|\alpha_{n}-\alpha_{n-1}\right|\right) \\
& \leq\left(1-\beta_{n}(\tau-\gamma \rho)\right)\left\|x_{n}-x_{n-1}\right\|+M\left(\left|\alpha_{n}-\alpha_{n-1}\right|+\left|\beta_{n}-\beta_{n-1}\right|\right. \\
& \left.+\left|\theta_{n}-\theta_{n-1}\right|+\left|r_{n}-r_{n-1}\right|\right)
\end{aligned}
$$

where $M_{1}+\left\|\gamma V y_{n}-\mu F T \tilde{u}_{n}\right\| \leq M, \forall n \geq 0$ for some $M \geq 0$. Therefore,

$$
\begin{aligned}
\frac{\left\|x_{n+1}-x_{n}\right\|}{\theta_{n}} & \left(1-\beta_{n}(\tau-\gamma \rho)\right) \frac{\left\|x_{n}-x_{n-1}\right\|}{\theta_{n}} \\
\leq & \quad M\left(\frac{\left|\alpha_{n}-\alpha_{n-1}\right|}{\theta_{n}}+\frac{\left|\beta_{n}-\beta_{n-1}\right|}{\theta_{n}}+\frac{\left|\theta_{n}-\theta_{n-1}\right|}{\theta_{n}}+\frac{\left|r_{n}-r_{n-1}\right|}{\theta_{n}}\right) \\
& \quad\left(1-(\tau-\gamma \rho) \beta_{n}\right) \frac{\| x_{n}-x_{n-1} \mid}{\theta_{n-1}}+\left(1-(\tau-\gamma \rho) \beta_{n}\right)\left\|x_{n}-x_{n-1}\right\|\left(\frac{1}{\theta_{n}}-\frac{1}{\theta_{n-1}}\right) \\
& +M\left(\frac{\left|\alpha_{n}-\alpha_{n-1}\right|}{\theta_{n}}+\frac{\left|\beta_{n}-\beta_{n-1}\right|}{\theta_{n}}+\frac{\left|\theta_{n}-\theta_{n-1}\right|}{\theta_{n}}+\frac{\left|r_{n}-r_{n-1}\right|}{\theta_{n}}\right) \\
\leq & \left(1-(\tau-\gamma \rho) \beta_{n}\right) \frac{\left\|x_{n}-x_{n-1}\right\|}{\theta_{n-1}}+(\tau-\gamma \rho) \beta_{n} \cdot \frac{1}{\tau-\gamma \rho}\left\{\left\|x_{n}-x_{n-1}\right\| \frac{1}{\beta_{n}}\left|\frac{1}{\theta_{n}}-\frac{1}{\theta_{n-1}}\right|\right. \\
& \left.+M\left(\frac{\left|\alpha_{n}-\alpha_{n-1}\right|}{\beta_{n} \theta_{n}}+\frac{\left|\beta_{n}-\beta_{n-1}\right|}{\beta_{n} \theta_{n}}+\frac{\left|\theta_{n}-\theta_{n-1}\right|}{\beta_{n} \theta_{n}}+\frac{\left|r_{n}-r_{n-1}\right|}{\beta_{n} \theta_{n}}\right)\right\}
\end{aligned}
$$




$$
\begin{aligned}
\leq & \left(1-(\tau-\gamma \rho) \beta_{n}\right) \frac{\left\|x_{n}-x_{n-1}\right\|}{\theta_{n-1}}+(\tau-\gamma \rho) \beta_{n} \cdot \frac{\tilde{M}}{\tau-\gamma \rho}\left\{\frac{1}{\beta_{n}}\left|\frac{1}{\theta_{n}}-\frac{1}{\theta_{n-1}}\right|\right. \\
& \left.+\frac{\left|\alpha_{n}-\alpha_{n-1}\right|}{\beta_{n} \theta_{n}}+\frac{1}{\theta_{n}}\left|1-\frac{\beta_{n-1}}{\beta_{n}}\right|+\frac{1}{\beta_{n}}\left|1-\frac{\theta_{n-1}}{\theta_{n}}\right|+\frac{\left|r_{n}-r_{n-1}\right|}{\beta_{n} \theta_{n}}\right\},
\end{aligned}
$$

where $M+\left\|x_{n}-x_{n-1}\right\| \leq \widetilde{M}, \forall n \geq 1$ for some $\widetilde{M} \geq 0$. From (H1), (H2), and (H4), it follows that $\sum_{n=0}^{\infty}(\tau-\gamma \rho) \beta_{n}=+\infty$ and

$$
\begin{aligned}
& \lim _{n \rightarrow \infty} \frac{\tilde{M}}{\tau-\gamma \rho}\left\{\frac{1}{\beta_{n}}\left|\frac{1}{\theta_{n}}-\frac{1}{\theta_{n-1}}\right|+\frac{\left|\alpha_{n}-\alpha_{n-1}\right|}{\beta_{n} \theta_{n}}+\frac{1}{\theta_{n}}\left|1-\frac{\beta_{n-1}}{\beta_{n}}\right|\right. \\
& \left.+\frac{1}{\beta_{n}}\left|1-\frac{\theta_{n-1}}{\theta_{n}}\right|+\frac{\left|r_{n}-r_{n-1}\right|}{\beta_{n} \theta_{n}}\right\}=0 .
\end{aligned}
$$

Applying Lemma 2.7 to (3.8), we immediately conclude that

$$
\lim _{n \rightarrow \infty} \frac{\left\|x_{n+1}-x_{n}\right\|}{\theta_{n}}=0 .
$$

In particular, from $(\mathrm{H} 3)$ it follows that

$$
\lim _{n \rightarrow \infty}\left\|x_{n+1}-x_{n}\right\|=0 .
$$

Step 2. $\lim _{n \rightarrow \infty}\left\|x_{n}-u_{n}\right\|=0$ and $\lim _{n \rightarrow \infty}\left\|y_{n}-\tilde{u}_{n}\right\|=0$.

By the firm nonexpansivity of $T_{r_{n}}$, if $v \in \operatorname{MEP}(\Theta, \varphi)=\operatorname{Fix}\left(T_{r_{n}}\right)$, we have

$$
\begin{aligned}
\left\|u_{n}-v\right\|^{2} & =\left\|T_{r_{n}} x_{n}-T_{r_{n}} v\right\|^{2} \\
& \leq\left\langle T_{r_{n}} x_{n}-T_{r_{n}} v, x_{n}-v\right\rangle \\
& =\frac{1}{2}\left\{\left\|u_{n}-v\right\|^{2}+\left\|x_{n}-v\right\|^{2}-\left\|T_{r_{n}} x_{n}-T_{r_{n}} v-\left(x_{n}-v\right)\right\|^{2}\right\} .
\end{aligned}
$$

This immediately yields

$$
\left\|u_{n}-v\right\|^{2} \leq\left\|x_{n}-v\right\|^{2}-\left\|x_{n}-u_{n}\right\|^{2}
$$

Let $p \in \operatorname{Fix}(T) \cap \operatorname{MEP}(\Theta, \varphi) \cap \Gamma$. We have

$$
\begin{aligned}
\left\|\tilde{u}_{n}-p\right\|= & \left\|P_{C}\left(I-\lambda \nabla f_{\alpha_{n}}\right) u_{n}-P_{C}(I-\lambda \nabla f) p\right\| \\
\leq & \left\|P_{C}\left(I-\lambda \nabla f_{\alpha_{n}}\right) u_{n}-P_{C}\left(I-\lambda \nabla f_{\alpha_{n}}\right) p\right\| \\
& +\left\|P_{C}\left(I-\lambda \nabla f_{\alpha_{n}}\right) p-P_{C}(I-\lambda \nabla f) p\right\| \\
\leq & \left\|u_{n}-p\right\|+\left\|P_{C}\left(I-\lambda \nabla f_{\alpha_{n}}\right) p-P_{C}(I-\lambda \nabla f) p\right\| \\
\leq & \left\|u_{n}-p\right\|+\lambda \alpha_{n}\|p\| .
\end{aligned}
$$

Note that

$$
\begin{aligned}
y_{n}-p & =\theta_{n} \gamma S x_{n}-\theta_{n} \mu F p+\left(I-\theta_{n} \mu F\right) \tilde{u}_{n}-\left(I-\theta_{n} \mu F\right) p \\
& =\theta_{n}\left(\gamma S x_{n}-\mu F p\right)+\left(1-\theta_{n}\right)\left(\tilde{u}_{n}-p\right)+\theta_{n}\left[(I-\mu F) \tilde{u}_{n}-(I-\mu F) p\right] \\
& =\theta_{n}\left(\gamma S x_{n}+(I-\mu F) \tilde{u}_{n}-p\right)+\left(1-\theta_{n}\right)\left(\tilde{u}_{n}-p\right) .
\end{aligned}
$$


Hence we have

$$
y_{n}-\tilde{u}_{n}=\theta_{n}\left(\gamma S x_{n}+(I-\mu F) \tilde{u}_{n}-\tilde{u}_{n}\right) .
$$

By Lemmas 2.10 and 2.12, we have from (3.9) and (3.10) that

$$
\begin{aligned}
\left\|y_{n}-p\right\|^{2} & \\
= & \left\|\theta_{n}\left(\gamma S x_{n}+(I-\mu F) \tilde{u}_{n}-p\right)+\left(1-\theta_{n}\right)\left(\tilde{u}_{n}-p\right)\right\|^{2} \\
= & \theta_{n}\left\|\gamma S x_{n}+(I-\mu F) \tilde{u}_{n}-p\right\|^{2}+\left(1-\theta_{n}\right)\left\|\tilde{u}_{n}-p\right\|^{2} \\
& \quad-\theta_{n}\left(1-\theta_{n}\right)\left\|\gamma S x_{n}+(I-\mu F) \tilde{u}_{n}-\tilde{u}_{n}\right\|^{2} \\
= & \theta_{n}\left\|\gamma S x_{n}+(I-\mu F) \tilde{u}_{n}-p\right\|^{2}+\left(1-\theta_{n}\right)\left\|\tilde{u}_{n}-p\right\|^{2}-\frac{1-\theta_{n}}{\theta_{n}}\left\|y_{n}-\tilde{u}_{n}\right\|^{2} \\
\leq & \theta_{n}\left\|\gamma S x_{n}+(I-\mu F) \tilde{u}_{n}-p\right\|^{2}+\left\|\tilde{u}_{n}-p\right\|^{2}-\frac{1-\theta_{n}}{\theta_{n}}\left\|y_{n}-\tilde{u}_{n}\right\|^{2} .
\end{aligned}
$$

Furthermore, utilizing Lemmas 2.11 and 2.12 we have from (3.9) and (3.10) that

$$
\begin{aligned}
& \left\|x_{n+1}-p\right\|^{2} \\
& =\left\|\beta_{n} \gamma V y_{n}-\beta_{n} \mu F T p+\left(I-\beta_{n} \mu F\right) T \tilde{u}_{n}-\left(I-\beta_{n} \mu F\right) T p\right\|^{2} \\
& \leq\left(\left\|\beta_{n} \gamma V y_{n}-\beta_{n} \mu F T p\right\|+\left\|\left(I-\beta_{n} \mu F\right) T \tilde{u}_{n}-\left(I-\beta_{n} \mu F\right) T p\right\|\right)^{2} \\
& \leq\left(\beta_{n}\left\|\gamma V y_{n}-\mu F p\right\|+\left(1-\beta_{n} \tau\right)\left\|\tilde{u}_{n}-p\right\|\right)^{2} \\
& \leq \beta_{n} \frac{1}{\tau}\left\|\gamma V y_{n}-\mu F p\right\|^{2}+\left(1-\beta_{n} \tau\right)\left\|\tilde{u}_{n}-p\right\|^{2} \\
& \leq \beta_{n} \frac{1}{\tau}\left[\left\|\gamma V y_{n}-\gamma V p\right\|^{2}+2\left\langle\gamma V p-\mu F p, \gamma V y_{n}-\mu F p\right\rangle\right]+\left(1-\beta_{n} \tau\right)\left\|\tilde{u}_{n}-p\right\|^{2} \\
& \leq \beta_{n} \frac{\gamma^{2} \rho^{2}}{\tau}\left\|y_{n}-p\right\|^{2}+\beta_{n} \frac{2}{\tau}\|\gamma V p-\mu F p\|\left\|\gamma V y_{n}-\mu F p\right\|+\left(1-\beta_{n} \tau\right)\left\|\tilde{u}_{n}-p\right\|^{2} \\
& \leq \beta_{n} \frac{\gamma^{2} \rho^{2}}{\tau}\left[\theta_{n}\left\|\gamma S x_{n}+(I-\mu F) \tilde{u}_{n}-p\right\|^{2}+\left\|\tilde{u}_{n}-p\right\|^{2}-\frac{1-\theta_{n}}{\theta_{n}}\left\|y_{n}-\tilde{u}_{n}\right\|^{2}\right] \\
& +\beta_{n} \frac{2}{\tau}\|\gamma V p-\mu F p\|\left\|\gamma V y_{n}-\mu F p\right\|+\left(1-\beta_{n} \tau\right)\left\|\tilde{u}_{n}-p\right\|^{2} \\
& =\left(1-\beta_{n}\left(\tau-\frac{\gamma^{2} \rho^{2}}{\tau}\right)\right)\left\|\tilde{u}_{n}-p\right\|^{2}+\beta_{n} \theta_{n} \frac{\gamma^{2} \rho^{2}}{\tau}\left\|\gamma S x_{n}+(I-\mu F) \tilde{u}_{n}-p\right\|^{2} \\
& -\frac{\beta_{n} \gamma^{2} \rho^{2}\left(1-\theta_{n}\right)}{\tau \theta_{n}}\left\|y_{n}-\tilde{u}_{n}\right\|^{2}+\beta_{n} \frac{2}{\tau}\|\gamma V p-\mu F p\|\left\|\gamma V y_{n}-\mu F p\right\| \\
& \leq\left\|\tilde{u}_{n}-p\right\|^{2}+\beta_{n} \theta_{n} \frac{\gamma^{2} \rho^{2}}{\tau}\left\|\gamma S x_{n}+(I-\mu F) \tilde{u}_{n}-p\right\|^{2} \\
& -\frac{\beta_{n} \gamma^{2} \rho^{2}\left(1-\theta_{n}\right)}{\tau \theta_{n}}\left\|y_{n}-\tilde{u}_{n}\right\|^{2}+\beta_{n} \frac{2}{\tau}\|\gamma V p-\mu F p\|\left\|\gamma V y_{n}-\mu F p\right\| \\
& \leq\left(\left\|u_{n}-p\right\|+\lambda \alpha_{n}\|p\|\right)^{2}+\beta_{n} \theta_{n} \frac{\gamma^{2} \rho^{2}}{\tau}\left\|\gamma S x_{n}+(I-\mu F) \tilde{u}_{n}-p\right\|^{2} \\
& -\frac{\beta_{n} \gamma^{2} \rho^{2}\left(1-\theta_{n}\right)}{\tau \theta_{n}}\left\|y_{n}-\tilde{u}_{n}\right\|^{2}+\beta_{n} \frac{2}{\tau}\|\gamma V p-\mu F p\|\left\|\gamma V y_{n}-\mu F p\right\|
\end{aligned}
$$




$$
\begin{aligned}
\leq & \left\|u_{n}-p\right\|^{2}+\lambda \alpha_{n}\|p\|\left(2\left\|u_{n}-p\right\|+\lambda \alpha_{n}\|p\|\right) \\
& +\beta_{n} \theta_{n} \frac{\gamma^{2} \rho^{2}}{\tau}\left\|\gamma S x_{n}+(I-\mu F) \tilde{u}_{n}-p\right\|^{2}-\frac{\beta_{n} \gamma^{2} \rho^{2}\left(1-\theta_{n}\right)}{\tau \theta_{n}}\left\|y_{n}-\tilde{u}_{n}\right\|^{2} \\
& +\beta_{n} \frac{2}{\tau}\|\gamma V p-\mu F p\|\left\|\gamma V y_{n}-\mu F p\right\| \\
\leq & \left\|x_{n}-p\right\|^{2}-\left\|x_{n}-u_{n}\right\|^{2}+\lambda \alpha_{n}\|p\|\left(2\left\|u_{n}-p\right\|+\lambda \alpha_{n}\|p\|\right) \\
& +\beta_{n} \theta_{n} \frac{\gamma^{2} \rho^{2}}{\tau}\left\|\gamma S x_{n}+(I-\mu F) \tilde{u}_{n}-p\right\|^{2}-\frac{\beta_{n} \gamma^{2} \rho^{2}\left(1-\theta_{n}\right)}{\tau \theta_{n}}\left\|y_{n}-\tilde{u}_{n}\right\|^{2} \\
& +\beta_{n} \frac{2}{\tau}\|\gamma V p-\mu F p\|\left\|\gamma V y_{n}-\mu F p\right\| .
\end{aligned}
$$

It turns out therefore that

$$
\begin{aligned}
\| x_{n}- & u_{n}\left\|^{2}+\frac{\beta_{n} \gamma^{2} \rho^{2}\left(1-\theta_{n}\right)}{\tau \theta_{n}}\right\| y_{n}-\tilde{u}_{n} \|^{2} \\
\leq & \left\|x_{n}-p\right\|^{2}-\left\|x_{n+1}-p\right\|^{2}+\lambda \alpha_{n}\|p\|\left(2\left\|u_{n}-p\right\|+\lambda \alpha_{n}\|p\|\right) \\
& +\beta_{n} \theta_{n} \frac{\gamma^{2} \rho^{2}}{\tau}\left\|\gamma S x_{n}+(I-\mu F) \tilde{u}_{n}-p\right\|^{2} \\
& +\beta_{n} \frac{2}{\tau}\|\gamma V p-\mu F p\|\left\|\gamma V y_{n}-\mu F p\right\| \\
\leq & \left(\left\|x_{n}-p\right\|+\left\|x_{n+1}-p\right\|\right)\left\|x_{n}-x_{n+1}\right\|+\lambda \alpha_{n}\|p\|\left(2\left\|u_{n}-p\right\|+\lambda \alpha_{n}\|p\|\right) \\
& +\beta_{n} \theta_{n} \frac{\gamma^{2} \rho^{2}}{\tau}\left\|\gamma S x_{n}+(I-\mu F) \tilde{u}_{n}-p\right\|^{2} \\
& +\beta_{n} \frac{2}{\tau}\|\gamma V p-\mu F p\|\left\|\gamma V y_{n}-\mu F p\right\| .
\end{aligned}
$$

Then it is clear that

$$
\begin{aligned}
\left\|x_{n}-u_{n}\right\|^{2} \leq & \left(\left\|x_{n}-p\right\|+\left\|x_{n+1}-p\right\|\right)\left\|x_{n}-x_{n+1}\right\|+\lambda \alpha_{n}\|p\|\left(2\left\|u_{n}-p\right\|+\lambda \alpha_{n}\|p\|\right) \\
& +\beta_{n} \theta_{n} \frac{\gamma^{2} \rho^{2}}{\tau}\left\|\gamma S x_{n}+(I-\mu F) \tilde{u}_{n}-p\right\|^{2} \\
& +\beta_{n} \frac{2}{\tau}\|\gamma V p-\mu F p\|\left\|\gamma V y_{n}-\mu F p\right\| .
\end{aligned}
$$

Since $\alpha_{n} \rightarrow 0, \beta_{n} \rightarrow 0, \theta_{n} \rightarrow 0$, and $\left\|x_{n+1}-x_{n}\right\| \rightarrow 0$, we conclude that

$$
\lim _{n \rightarrow \infty}\left\|x_{n}-u_{n}\right\|=0
$$

Furthermore,

$$
\begin{aligned}
& \frac{\beta_{n} \gamma^{2} \rho^{2}\left(1-\theta_{n}\right)}{\tau \theta_{n}}\left\|y_{n}-\tilde{u}_{n}\right\|^{2} \\
& \leq\left(\left\|x_{n}-p\right\|+\left\|x_{n+1}-p\right\|\right)\left\|x_{n}-x_{n+1}\right\|+\lambda \alpha_{n}\|p\|\left(2\left\|u_{n}-p\right\|+\lambda \alpha_{n}\|p\|\right) \\
& \quad+\beta_{n} \theta_{n} \frac{\gamma^{2} \rho^{2}}{\tau}\left\|\gamma S x_{n}+(I-\mu F) \tilde{u}_{n}-p\right\|^{2}+\beta_{n} \frac{2}{\tau}\|\gamma V p-\mu F p\|\left\|\gamma V y_{n}-\mu F p\right\| .
\end{aligned}
$$


This yields

$$
\begin{aligned}
& \frac{\gamma^{2} \rho^{2}\left(1-\theta_{n}\right)}{\tau}\left\|y_{n}-\tilde{u}_{n}\right\|^{2} \\
& \leq\left(\left\|x_{n}-p\right\|+\left\|x_{n+1}-p\right\|\right) \frac{\theta_{n}}{\beta_{n}}\left\|x_{n}-x_{n+1}\right\|+\frac{\alpha_{n} \theta_{n}}{\beta_{n}} \lambda\|p\|\left(2\left\|u_{n}-p\right\|+\lambda \alpha_{n}\|p\|\right) \\
& \quad+\theta_{n}^{2} \frac{\gamma^{2} \rho^{2}}{\tau}\left\|\gamma S x_{n}+(I-\mu F) \tilde{u}_{n}-p\right\|^{2}+\theta_{n} \frac{2}{\tau}\|\gamma V p-\mu F p\|\left\|\gamma V y_{n}-\mu F p\right\| .
\end{aligned}
$$

Since $\frac{\left\|x_{n+1}-x_{n}\right\|}{\theta_{n}} \rightarrow 0, \frac{\alpha_{n}+\beta_{n}}{\theta_{n}} \rightarrow 0$, and $\frac{\theta_{n}^{2}}{\beta_{n}} \rightarrow 0$ as $n \rightarrow \infty$, we have

$$
\lim _{n \rightarrow \infty} \frac{\theta_{n}}{\beta_{n}}\left\|x_{n}-x_{n+1}\right\|=\lim _{n \rightarrow \infty} \frac{\left\|x_{n}-x_{n+1}\right\|}{\theta_{n}} \cdot \frac{\theta_{n}^{2}}{\beta_{n}}=0
$$

and

$$
\lim _{n \rightarrow \infty} \frac{\alpha_{n} \theta_{n}}{\beta_{n}}=\lim _{n \rightarrow \infty} \frac{\alpha_{n}}{\theta_{n}} \cdot \frac{\theta_{n}^{2}}{\beta_{n}}=0 .
$$

Therefore, from the last inequality we have

$$
\lim _{n \rightarrow \infty}\left\|y_{n}-\tilde{u}_{n}\right\|=0
$$

Step 3. $\lim _{n \rightarrow \infty}\left\|u_{n}-\tilde{u}_{n}\right\|=0$ and $\lim _{n \rightarrow \infty}\left\|x_{n}-y_{n}\right\|=0$.

Let $p \in \operatorname{Fix}(T) \cap \operatorname{Fix}(\Gamma) \cap \Xi$. Utilizing Lemmas 2.6 and 2.11 we have from (3.12) that

$$
\begin{aligned}
\left\|x_{n+1}-p\right\|^{2} & \\
\leq & \left\|\tilde{u}_{n}-p\right\|^{2}+\beta_{n} \theta_{n} \frac{\gamma^{2} \rho^{2}}{\tau}\left\|\gamma S x_{n}+(I-\mu F) \tilde{u}_{n}-p\right\|^{2} \\
& -\frac{\beta_{n} \gamma^{2} \rho^{2}\left(1-\theta_{n}\right)}{\tau \theta_{n}}\left\|y_{n}-\tilde{u}_{n}\right\|^{2}+\beta_{n} \frac{2}{\tau}\|\gamma V p-\mu F p\|\left\|\gamma V y_{n}-\mu F p\right\| \\
\leq & \left\|P_{C}\left(I-\lambda \nabla f_{\alpha_{n}}\right) u_{n}-P_{C}(I-\lambda \nabla f) p\right\|^{2}+\beta_{n} \theta_{n} \frac{\gamma^{2} \rho^{2}}{\tau}\left\|\gamma S x_{n}+(I-\mu F) \tilde{u}_{n}-p\right\|^{2} \\
& +\beta_{n} \frac{2}{\tau}\|\gamma V p-\mu F p\|\left\|\gamma V y_{n}-\mu F p\right\| \\
\leq & \left\|(I-\lambda \nabla f) u_{n}-(I-\lambda \nabla f) p-\lambda \alpha_{n} u_{n}\right\|^{2}+\beta_{n} \theta_{n} \frac{\gamma^{2} \rho^{2}}{\tau}\left\|\gamma S x_{n}+(I-\mu F) \tilde{u}_{n}-p\right\|^{2} \\
& +\beta_{n} \frac{2}{\tau}\|\gamma V p-\mu F p\|\left\|\gamma V y_{n}-\mu F p\right\| \\
\leq & \left\|(I-\lambda \nabla f) u_{n}-(I-\lambda \nabla f) p\right\|^{2}-2 \lambda \alpha_{n}\left\langle u_{n},\left(I-\lambda \nabla f_{\alpha_{n}}\right) u_{n}-(I-\lambda \nabla f) p\right\rangle \\
& +\beta_{n} \theta_{n} \frac{\gamma^{2} \rho^{2}}{\tau}\left\|\gamma S x_{n}+(I-\mu F) \tilde{u}_{n}-p\right\|^{2}+\beta_{n} \frac{2}{\tau}\|\gamma V p-\mu F p\|\left\|\gamma V y_{n}-\mu F p\right\| \\
& +\beta_{n} \theta_{n} \frac{\gamma^{2} \rho^{2}}{\tau}\left\|\gamma S x_{n}+(I-\mu F) \tilde{u}_{n}-p\right\|^{2}+\beta_{n} \frac{2}{\tau}\|\gamma V p-\mu F p\|\left\|\gamma V y_{n}-\mu F p\right\| \\
\leq & \left\|u_{n}-p\right\|^{2}+\lambda\left(\lambda-\frac{2}{L}\right)\left\|\nabla f\left(u_{n}\right)-\nabla f(p)\right\|^{2} \\
& +2 \lambda \alpha_{n}\left\|u_{n}\right\|\left\|\left(I-\lambda \nabla f_{\alpha_{n}}\right) u_{n}-(I-\lambda \nabla f) p\right\|
\end{aligned}
$$




$$
\begin{aligned}
\leq & \left\|x_{n}-p\right\|^{2}+\lambda\left(\lambda-\frac{2}{L}\right)\left\|\nabla f\left(u_{n}\right)-\nabla f(p)\right\|^{2} \\
& +2 \lambda \alpha_{n}\left\|u_{n}\right\|\left\|\left(I-\lambda \nabla f_{\alpha_{n}}\right) u_{n}-(I-\lambda \nabla f) p\right\| \\
& +\beta_{n} \theta_{n} \frac{\gamma^{2} \rho^{2}}{\tau}\left\|\gamma S x_{n}+(I-\mu F) \tilde{u}_{n}-p\right\|^{2}+\beta_{n} \frac{2}{\tau}\|\gamma V p-\mu F p\|\left\|\gamma V y_{n}-\mu F p\right\| .
\end{aligned}
$$

Hence,

$$
\begin{aligned}
& \lambda\left(\frac{2}{L}-\lambda\right)\left\|\nabla f\left(u_{n}\right)-\nabla f(p)\right\|^{2} \\
& \leq\left\|x_{n}-p\right\|^{2}-\left\|x_{n+1}-p\right\|^{2}+2 \lambda \alpha_{n}\left\|u_{n}\right\|\left\|\left(I-\lambda \nabla f_{\alpha_{n}}\right) u_{n}-(I-\lambda \nabla f) p\right\| \\
& \quad+\beta_{n} \theta_{n} \frac{\gamma^{2} \rho^{2}}{\tau}\left\|\gamma S x_{n}+(I-\mu F) \tilde{u}_{n}-p\right\|^{2}+\beta_{n} \frac{2}{\tau}\|\gamma V p-\mu F p\|\left\|\gamma V y_{n}-\mu F p\right\| \\
& \leq\left(\left\|x_{n}-p\right\|+\left\|x_{n+1}-p\right\|\right)\left\|x_{n}-x_{n+1}\right\|+2 \lambda \alpha_{n}\left\|u_{n}\right\|\left\|\left(I-\lambda \nabla f_{\alpha_{n}}\right) u_{n}-(I-\lambda \nabla f) p\right\| \\
& \quad+\beta_{n} \theta_{n} \frac{\gamma^{2} \rho^{2}}{\tau}\left\|\gamma S x_{n}+(I-\mu F) \tilde{u}_{n}-p\right\|^{2}+\beta_{n} \frac{2}{\tau}\|\gamma V p-\mu F p\|\left\|\gamma V y_{n}-\mu F p\right\| .
\end{aligned}
$$

Since $\alpha_{n} \rightarrow 0, \beta_{n} \rightarrow 0, \theta_{n} \rightarrow 0$, and $\left\|x_{n+1}-x_{n}\right\| \rightarrow 0$, it follows from $0<\lambda<\frac{2}{L}$ that $\lim _{n \rightarrow \infty}\left\|\nabla f\left(u_{n}\right)-\nabla f(p)\right\|=0$, and hence

$$
\lim _{n \rightarrow \infty}\left\|\nabla f_{\alpha_{n}}\left(u_{n}\right)-\nabla f(p)\right\|=0 .
$$

Furthermore, from the firm nonexpansiveness of $P_{C}$ we obtain

$$
\begin{aligned}
\left\|\tilde{u}_{n}-p\right\|^{2}= & \left\|P_{C}\left(I-\lambda \nabla f_{\alpha_{n}}\right) u_{n}-P_{C}(I-\lambda \nabla f) p\right\|^{2} \\
\leq & \left\langle\left(I-\lambda \nabla f_{\alpha_{n}}\right) u_{n}-(I-\lambda \nabla f) p, \tilde{u}_{n}-p\right\rangle \\
= & \frac{1}{2}\left\{\left\|\left(I-\lambda \nabla f_{\alpha_{n}}\right) u_{n}-(I-\lambda \nabla f) p\right\|^{2}+\left\|\tilde{u}_{n}-p\right\|^{2}\right. \\
& \left.-\left\|\left(I-\lambda \nabla f_{\alpha_{n}}\right) u_{n}-(I-\lambda \nabla f) p-\left(\tilde{u}_{n}-p\right)\right\|^{2}\right\} \\
\leq & \frac{1}{2}\left\{\left\|u_{n}-p\right\|^{2}+2 \lambda\left\|\nabla f_{\alpha_{n}}\left(u_{n}\right)-\nabla f(p)\right\|\left\|\left(I-\lambda \nabla f_{\alpha_{n}}\right) u_{n}-(I-\lambda \nabla f) p\right\|\right. \\
& +\left\|\tilde{u}_{n}-p\right\|^{2}-\left\|u_{n}-\tilde{u}_{n}\right\|^{2}+2 \lambda\left\langle u_{n}-\tilde{u}_{n}, \nabla f_{\alpha_{n}}\left(u_{n}\right)-\nabla f(p)\right\rangle \\
& \left.-\lambda^{2}\left\|\nabla f_{\alpha_{n}}\left(u_{n}\right)-\nabla f(p)\right\|^{2}\right\} .
\end{aligned}
$$

Consequently,

$$
\begin{aligned}
& \left\|\tilde{u}_{n}-p\right\|^{2} \\
& \quad \leq\left\|u_{n}-p\right\|^{2}-\left\|u_{n}-\tilde{u}_{n}\right\|^{2}+2 \lambda\left\|\nabla f_{\alpha_{n}}\left(u_{n}\right)-\nabla f(p)\right\|\left\|\left(I-\lambda \nabla f_{\alpha_{n}}\right) u_{n}-(I-\lambda \nabla f) p\right\| \\
& \quad+2 \lambda\left\langle u_{n}-\tilde{u}_{n}, \nabla f_{\alpha_{n}}\left(u_{n}\right)-\nabla f(p)\right\rangle-\lambda^{2}\left\|\nabla f_{\alpha_{n}}\left(u_{n}\right)-\nabla f(p)\right\|^{2} .
\end{aligned}
$$

Thus, from (3.12) we have

$$
\begin{aligned}
& \left\|x_{n+1}-p\right\|^{2} \\
& \quad \leq\left\|\tilde{u}_{n}-p\right\|^{2}+\beta_{n} \theta_{n} \frac{\gamma^{2} \rho^{2}}{\tau}\left\|\gamma S x_{n}+(I-\mu F) \tilde{u}_{n}-p\right\|^{2}
\end{aligned}
$$




$$
\begin{aligned}
& -\frac{\beta_{n} \gamma^{2} \rho^{2}\left(1-\theta_{n}\right)}{\tau \theta_{n}}\left\|y_{n}-\tilde{u}_{n}\right\|^{2}+\beta_{n} \frac{2}{\tau}\|\gamma V p-\mu F p\|\left\|\gamma V y_{n}-\mu F p\right\| \\
\leq & \left\|u_{n}-p\right\|^{2}-\left\|u_{n}-\tilde{u}_{n}\right\|^{2}+2 \lambda\left\|\nabla f_{\alpha_{n}}\left(u_{n}\right)-\nabla f(p)\right\|\left\|\left(I-\lambda \nabla f_{\alpha_{n}}\right) u_{n}-(I-\lambda \nabla f) p\right\| \\
& +2 \lambda\left\langle u_{n}-\tilde{u}_{n}, \nabla f_{\alpha_{n}}\left(u_{n}\right)-\nabla f(p)\right\rangle-\lambda^{2}\left\|\nabla f_{\alpha_{n}}\left(u_{n}\right)-\nabla f(p)\right\|^{2} \\
& +\beta_{n} \theta_{n} \frac{\gamma^{2} \rho^{2}}{\tau}\left\|\gamma S x_{n}+(I-\mu F) \tilde{u}_{n}-p\right\|^{2}-\frac{\beta_{n} \gamma^{2} \rho^{2}\left(1-\theta_{n}\right)}{\tau \theta_{n}}\left\|y_{n}-\tilde{u}_{n}\right\|^{2} \\
& +\beta_{n} \frac{2}{\tau}\|\gamma V p-\mu F p\|\left\|\gamma V y_{n}-\mu F p\right\| \\
\leq & \left\|x_{n}-p\right\|^{2}-\left\|u_{n}-\tilde{u}_{n}\right\|^{2}+2 \lambda\left\|\nabla f_{\alpha_{n}}\left(u_{n}\right)-\nabla f(p)\right\|\left\|\left(I-\lambda \nabla f_{\alpha_{n}}\right) u_{n}-(I-\lambda \nabla f) p\right\| \\
& +2 \lambda\left\|u_{n}-\tilde{u}_{n}\right\|\left\|\nabla f_{\alpha_{n}}\left(u_{n}\right)-\nabla f(p)\right\|+\beta_{n} \theta_{n} \frac{\gamma^{2} \rho^{2}}{\tau}\left\|\gamma S x_{n}+(I-\mu F) \tilde{u}_{n}-p\right\|^{2} \\
& +\beta_{n} \frac{2}{\tau}\|\gamma V p-\mu F p\|\left\|\gamma V y_{n}-\mu F p\right\| .
\end{aligned}
$$

This implies that

$$
\begin{aligned}
\| u_{n}- & \tilde{u}_{n} \|^{2} \\
\leq & \left\|x_{n}-p\right\|^{2}-\left\|x_{n+1}-p\right\|^{2}+2 \lambda\left\|\nabla f_{\alpha_{n}}\left(u_{n}\right)-\nabla f(p)\right\|\left\|\left(I-\lambda \nabla f_{\alpha_{n}}\right) u_{n}-(I-\lambda \nabla f) p\right\| \\
& +2 \lambda\left\|u_{n}-\tilde{u}_{n}\right\|\left\|\nabla f_{\alpha_{n}}\left(u_{n}\right)-\nabla f(p)\right\|+\beta_{n} \theta_{n} \frac{\gamma^{2} \rho^{2}}{\tau}\left\|\gamma S x_{n}+(I-\mu F) \tilde{u}_{n}-p\right\|^{2} \\
& +\beta_{n} \frac{2}{\tau}\|\gamma V p-\mu F p\|\left\|\gamma V y_{n}-\mu F p\right\| \\
\leq & \left(\left\|x_{n}-p\right\|+\left\|x_{n+1}-p\right\|\right)\left\|x_{n}-x_{n+1}\right\| \\
& +2 \lambda\left\|\nabla f_{\alpha_{n}}\left(u_{n}\right)-\nabla f(p)\right\|\left\|\left(I-\lambda \nabla f_{\alpha_{n}}\right) u_{n}-(I-\lambda \nabla f) p\right\| \\
& +2 \lambda\left\|u_{n}-\tilde{u}_{n}\right\|\left\|\nabla f_{\alpha_{n}}\left(u_{n}\right)-\nabla f(p)\right\|+\beta_{n} \theta_{n} \frac{\gamma^{2} \rho^{2}}{\tau}\left\|\gamma S x_{n}+(I-\mu F) \tilde{u}_{n}-p\right\|^{2} \\
& +\beta_{n} \frac{2}{\tau}\|\gamma V p-\mu F p\|\left\|\gamma V y_{n}-\mu F p\right\| .
\end{aligned}
$$

Since $\theta_{n} \rightarrow 0, \beta_{n} \rightarrow 0,\left\|x_{n}-x_{n+1}\right\| \rightarrow 0$, and $\left\|\nabla f_{\alpha_{n}}\left(u_{n}\right)-\nabla f(p)\right\| \rightarrow 0$, it follows that

$$
\lim _{n \rightarrow \infty}\left\|u_{n}-\tilde{u}_{n}\right\|=0
$$

This, together with $\left\|x_{n}-u_{n}\right\| \rightarrow 0$ and $\left\|y_{n}-\tilde{u}_{n}\right\| \rightarrow 0$ (due to Step 2), implies that

$$
\left\|x_{n}-y_{n}\right\| \leq\left\|x_{n}-u_{n}\right\|+\left\|u_{n}-\tilde{u}_{n}\right\|+\left\|\tilde{u}_{n}-y_{n}\right\| \rightarrow 0 \quad \text { as } n \rightarrow \infty
$$

and thus

$$
\lim _{n \rightarrow \infty}\left\|x_{n}-y_{n}\right\|=0
$$

Step 4. $\omega_{w}\left(x_{n}\right) \subset \operatorname{Fix}(T) \cap \operatorname{MEP}(\Theta, \varphi) \cap \Gamma$; moreover, if $\left\|x_{n}-y_{n}\right\|=o\left(\theta_{n}\right)$ in addition, then $\omega_{w}\left(x_{n}\right) \subset \Xi$. 
Let $p^{*} \in \omega_{w}\left(x_{n}\right)$. Then there exists a subsequence $\left\{x_{n_{i}}\right\}$ of $\left\{x_{n}\right\}$ such that $x_{n_{i}} \rightarrow p^{*}$. Since

$$
\begin{aligned}
x_{n+1}-\tilde{u}_{n} & =\beta_{n} \gamma S y_{n}+\left(I-\beta_{n} \mu F\right) T \tilde{u}_{n}-\tilde{u}_{n} \\
& =\beta_{n}\left(\gamma S y_{n}-\mu F T \tilde{u}_{n}\right)+T \tilde{u}_{n}-\tilde{u}_{n},
\end{aligned}
$$

we have

$$
\begin{aligned}
\left\|T \tilde{u}_{n}-\tilde{u}_{n}\right\| & =\left\|x_{n+1}-\tilde{u}_{n}-\beta_{n}\left(\gamma S y_{n}-\mu F T \tilde{u}_{n}\right)\right\| \\
& \leq\left\|x_{n+1}-\tilde{u}_{n}\right\|+\beta_{n}\left\|\gamma S y_{n}-\mu F T \tilde{u}_{n}\right\| \\
& \leq\left\|x_{n+1}-x_{n}\right\|+\left\|x_{n}-u_{n}\right\|+\left\|u_{n}-\tilde{u}_{n}\right\|+\beta_{n}\left\|\gamma S y_{n}-\mu F T \tilde{u}_{n}\right\| .
\end{aligned}
$$

Hence from $\left\|x_{n+1}-x_{n}\right\| \rightarrow 0,\left\|x_{n}-u_{n}\right\| \rightarrow 0,\left\|u_{n}-\tilde{u}_{n}\right\| \rightarrow 0$, and $\beta_{n} \rightarrow 0$, we get

$$
\lim _{n \rightarrow \infty}\left\|T \tilde{u}_{n}-\tilde{u}_{n}\right\|=0
$$

Since $\left\|x_{n}-u_{n}\right\| \rightarrow 0$ and $\left\|u_{n}-\tilde{u}_{n}\right\| \rightarrow 0$, we have $\tilde{u}_{n_{i}} \rightarrow p^{*}$. Utilizing Lemma 2.8 we derive $p^{*} \in \operatorname{Fix}(T)$.

Let us show that $p^{*} \in \operatorname{MEP}(\Theta, \varphi)$. As a matter of fact, since $u_{n}=T_{r_{n}} x_{n}$, for any $y \in C$ we have

$$
\Theta\left(u_{n}, y\right)+\varphi(y)-\varphi\left(u_{n}\right)+\frac{1}{r_{n}}\left\langle y-u_{n}, u_{n}-x_{n}\right\rangle \geq 0 .
$$

It follows from (A2) that

$$
\varphi(y)-\varphi\left(u_{n}\right)+\frac{1}{r_{n}}\left\langle y-u_{n}, u_{n}-x_{n}\right\rangle \geq \Theta\left(y, u_{n}\right) .
$$

Replacing $n$ by $n_{i}$, we have

$$
\varphi(y)-\varphi\left(u_{n_{i}}\right)+\frac{1}{r_{n_{i}}}\left\langle y-u_{n_{i}}, u_{n_{i}}-x_{n_{i}}\right\rangle \geq \Theta\left(y, u_{n_{i}}\right) .
$$

Since $\frac{u_{n_{i}}-x_{n_{i}}}{r_{n_{i}}} \rightarrow 0$ and $u_{n_{i}} \rightarrow p^{*}$, it follows from (A4) that

$$
0 \geq-\varphi(y)+\varphi\left(p^{*}\right)+\Theta\left(y, p^{*}\right), \quad \forall y \in C .
$$

Put $z_{t}=t y+(1-t) p^{*}$ for all $t \in(0,1]$ and $y \in C$. We have $z_{t} \in C$ and

$$
0 \geq-\varphi\left(z_{t}\right)+\varphi\left(p^{*}\right)+\Theta\left(z_{t}, p^{*}\right) .
$$

Utilizing (A1), (A4), and (3.13), we have

$$
\begin{aligned}
0 & =\Theta\left(z_{t}, z_{t}\right)+\varphi\left(z_{t}\right)-\varphi\left(z_{t}\right) \\
& \leq t \Theta\left(z_{t}, y\right)+(1-t) \Theta\left(z_{t}, p^{*}\right)+t \varphi(y)+(1-t) \varphi\left(p^{*}\right)-\varphi\left(z_{t}\right) \\
& \leq t\left(\Theta\left(z_{t}, y\right)+\varphi(y)-\varphi\left(z_{t}\right)\right)+(1-t)\left(\Theta\left(z_{t}, p^{*}\right)+\varphi\left(p^{*}\right)-\varphi\left(z_{t}\right)\right) \\
& \leq t\left(\Theta\left(z_{t}, y\right)+\varphi(y)-\varphi\left(z_{t}\right)\right),
\end{aligned}
$$


and hence

$$
0 \leq \Theta\left(z_{t}, y\right)+\varphi(y)-\varphi\left(z_{t}\right)
$$

Letting $t \rightarrow 0$ in (3.14) and utilizing (A3), we get, for each $y \in C$,

$$
0 \leq \Theta\left(p^{*}, y\right)+\varphi(y)-\varphi\left(p^{*}\right)
$$

Hence, $p^{*} \in \operatorname{MEP}(\Theta, \varphi)$.

Let us show that $p^{*} \in \Gamma$. From $\left\|x_{n}-u_{n}\right\| \rightarrow 0$ and $\left\|u_{n}-\tilde{u}_{n}\right\| \rightarrow 0$, we know that $u_{n_{i}} \rightarrow p^{*}$ and $\tilde{u}_{n_{i}} \rightarrow p^{*}$. Define

$$
\widetilde{T} v= \begin{cases}\nabla f(v)+N_{C} v, & \text { if } v \in C, \\ \emptyset, & \text { if } v \notin C,\end{cases}
$$

where

$$
N_{C} v=\{w \in H:\langle v-u, w\rangle \geq 0, \forall u \in C\} .
$$

Then $\widetilde{T}$ is maximal monotone and $0 \in \widetilde{T} v$ if and only if $v \in \operatorname{VI}(C, \nabla f)$; see [28] for more details. Let $(v, w) \in \operatorname{Gph}(\widetilde{T})$. Then we have

$$
w \in \widetilde{T} v=\nabla f(v)+N_{C} v
$$

and hence,

$$
w-\nabla f(v) \in N_{C} v
$$

Therefore,

$$
\langle v-u, w-\nabla f(v)\rangle \geq 0, \quad \forall u \in C
$$

On the other hand, from

$$
\tilde{u}_{n}=P_{C}\left(u_{n}-\lambda \nabla f_{\alpha_{n}}\left(u_{n}\right)\right) \text { and } v \in C \text {, }
$$

we have

$$
\left\langle u_{n}-\lambda \nabla f_{\alpha_{n}}\left(u_{n}\right)-\tilde{u}_{n}, \tilde{u}_{n}-v\right\rangle \geq 0,
$$

and hence

$$
\left\langle\nu-\tilde{u}_{n}, \frac{\tilde{u}_{n}-u_{n}}{\lambda}+\nabla f_{\alpha_{n}}\left(u_{n}\right)\right\rangle \geq 0 .
$$

Therefore, from

$$
w-\nabla f(v) \in N_{C}(v) \quad \text { and } \quad \tilde{u}_{n_{i}} \in C
$$


we have

$$
\begin{aligned}
\left\langle v-\tilde{u}_{n_{i}}, w\right\rangle \geq & \left\langle v-\tilde{u}_{n_{i}}, \nabla f(v)\right\rangle \\
\geq & \left\langle v-\tilde{u}_{n_{i}}, \nabla f(v)\right\rangle-\left\langle v-\tilde{u}_{n_{i}}, \frac{\tilde{u}_{n_{i}}-u_{n_{i}}}{\lambda}+\nabla f_{\alpha_{n_{i}}}\left(u_{n_{i}}\right)\right\rangle \\
= & \left\langle v-\tilde{u}_{n_{i}}, \nabla f(v)\right\rangle-\left\langle v-\tilde{u}_{n_{i}}, \frac{\tilde{u}_{n_{i}}-u_{n_{i}}}{\lambda}+\nabla f\left(u_{n_{i}}\right)\right\rangle-\alpha_{n_{i}}\left\langle v-\tilde{u}_{n_{i}}, u_{n_{i}}\right\rangle \\
= & \left\langle v-\tilde{u}_{n_{i}}, \nabla f(v)-\nabla f\left(\tilde{u}_{n_{i}}\right)\right\rangle+\left\langle v-\tilde{u}_{n_{i}}, \nabla f\left(\tilde{u}_{n_{i}}\right)-\nabla f\left(u_{n_{i}}\right)\right\rangle \\
& -\left\langle v-\tilde{u}_{n_{i}}, \frac{\tilde{u}_{n_{i}}-u_{n_{i}}}{\lambda}\right\rangle-\alpha_{n_{i}}\left\langle v-\tilde{u}_{n_{i}}, u_{n_{i}}\right\rangle \\
\geq & \left\langle v-\tilde{u}_{n_{i}}, \nabla f\left(\tilde{u}_{n_{i}}\right)-\nabla f\left(u_{n_{i}}\right)\right\rangle-\left\langle v-\tilde{u}_{n_{i}}, \frac{\tilde{u}_{n_{i}}-u_{n_{i}}}{\lambda}\right\rangle-\alpha_{n_{i}}\left\langle v-\tilde{u}_{n_{i}}, u_{n_{i}}\right\rangle .
\end{aligned}
$$

Hence, we obtain

$$
\left\langle v-p^{*}, w\right\rangle \geq 0 \quad \text { as } i \rightarrow \infty .
$$

Since $\widetilde{T}$ is maximal monotone, we have $p^{*} \in \widetilde{T}^{-1} 0$, and hence, $p^{*} \in \operatorname{VI}(C, \nabla f)$, which leads to $p^{*} \in \Gamma$. Consequently, $p^{*} \in \operatorname{Fix}(T) \cap \operatorname{MEP}(\Theta, \varphi) \cap \Gamma$. This shows that $\omega_{w}\left(x_{n}\right) \subset \operatorname{Fix}(T) \cap$ $\operatorname{MEP}(\Theta, \varphi) \cap \Gamma$.

Utilizing Lemmas 2.11 and 2.12, we have for every $p \in \operatorname{Fix}(T) \cap \operatorname{MEP}(\Theta, \varphi) \cap \Gamma$,

$$
\begin{aligned}
\| y_{n} & -p \|^{2} \\
& =\left\|\theta_{n} \gamma S x_{n}+(I-\mu F) \tilde{u}_{n}-p\right\|^{2} \\
& =\left\|\theta_{n}(\gamma S p-\mu F p)+\theta_{n}\left(\gamma S x_{n}-\gamma S p\right)+\left(I-\theta_{n} \mu F\right) \tilde{u}_{n}-\left(I-\theta_{n} \mu F\right) p\right\|^{2} \\
& \leq\left\|\theta_{n}\left(\gamma S x_{n}-\gamma S p\right)+\left(I-\theta_{n} \mu F\right) \tilde{u}_{n}-\left(I-\theta_{n} \mu F\right) p\right\|^{2}+2 \theta_{n}\left\langle\gamma S p-\mu F p, y_{n}-p\right\rangle \\
& \leq\left[\left\|\theta_{n}\left(\gamma S x_{n}-\gamma S p\right)\right\|+\left\|\left(I-\theta_{n} \mu F\right) \tilde{u}_{n}-\left(I-\theta_{n} \mu F\right) p\right\|\right]^{2}+2 \theta_{n}\left\langle\gamma S p-\mu F p, y_{n}-p\right\rangle \\
& \leq\left[\theta_{n} \gamma\left\|x_{n}-p\right\|+\left(1-\theta_{n} \tau\right)\left\|\tilde{u}_{n}-p\right\|\right]^{2}+2 \theta_{n}\left\langle\gamma S p-\mu F p, y_{n}-p\right\rangle \\
& \leq\left[\theta_{n} \gamma\left\|x_{n}-p\right\|+\left(1-\theta_{n} \tau\right)\left(\left\|u_{n}-p\right\|+\lambda \alpha_{n}\|p\|\right)\right]^{2}+2 \theta_{n}\left\langle\gamma S p-\mu F p, y_{n}-p\right\rangle \\
& \leq\left[\theta_{n} \gamma\left\|x_{n}-p\right\|+\left(1-\theta_{n} \tau\right)\left(\left\|x_{n}-p\right\|+\lambda \alpha_{n}\|p\|\right)\right]^{2}+2 \theta_{n}\left\langle\gamma S p-\mu F p, y_{n}-p\right\rangle \\
& \leq\left[\left(1-\theta_{n}(\tau-\gamma)\right)\left\|x_{n}-p\right\|+\lambda \alpha_{n}\|p\|\right]^{2}+2 \theta_{n}\left\langle\gamma S p-\mu F p, y_{n}-p\right\rangle \\
& \leq\left(\left\|x_{n}-p\right\|+\lambda \alpha_{n}\|p\|\right)^{2}+2 \theta_{n}\left\langle\gamma S p-\mu F p, y_{n}-p\right\rangle .
\end{aligned}
$$

Suppose now that $\left\|x_{n}-y_{n}\right\|=o\left(\theta_{n}\right)$ in addition. It follows from (3.15) that

$$
\begin{aligned}
& 2\left\langle\gamma S p-\mu F p, y_{n}-p\right\rangle \\
& \quad \leq \frac{1}{\theta_{n}}\left[\left(\left\|x_{n}-p\right\|+\lambda \alpha_{n}\|p\|\right)^{2}-\left\|y_{n}-p\right\|^{2}\right] \\
& \quad \leq \frac{1}{\theta_{n}}\left(\left\|x_{n}-p\right\|+\lambda \alpha_{n}\|p\|+\left\|y_{n}-p\right\|\right)\left(\left\|x_{n}-p\right\|+\lambda \alpha_{n}\|p\|-\left\|y_{n}-p\right\|\right) \\
& \quad \leq \frac{1}{\theta_{n}}\left(\left\|x_{n}-p\right\|+\lambda \alpha_{n}\|p\|+\left\|y_{n}-p\right\|\right)\left(\left\|x_{n}-y_{n}\right\|+\lambda \alpha_{n}\|p\|\right) .
\end{aligned}
$$


This, together with $\frac{\alpha_{n}}{\theta_{n}} \rightarrow 0$ and $\frac{\left\|x_{n}-y_{n}\right\|}{\theta_{n}} \rightarrow 0$, leads to

$$
\limsup _{n \rightarrow \infty}\left\langle\gamma S p-\mu F p, y_{n}-p\right\rangle \leq 0 \text {. }
$$

Observe that

$$
\begin{aligned}
& \limsup _{n \rightarrow \infty}\left\langle\gamma S p-\mu F p, x_{n}-p\right\rangle \\
& \quad=\limsup _{n \rightarrow \infty}\left(\left\langle\gamma S p-\mu F p, x_{n}-y_{n}\right\rangle+\left\langle\gamma S p-\mu F p, y_{n}-p\right\rangle\right) \\
& \quad=\limsup _{n \rightarrow \infty}\left\langle\gamma S p-\mu F p, y_{n}-p\right\rangle \leq 0 .
\end{aligned}
$$

So, it follows from $x_{n_{i}} \rightarrow p^{*}$ that

$$
\left\langle\gamma S p-\mu F p, p^{*}-p\right\rangle \leq 0, \quad \forall p \in \operatorname{Fix}(T) \cap \operatorname{MEP}(\Theta, \varphi) \cap \Gamma .
$$

Note also that $0<\gamma \leq \tau$ and

$$
\begin{aligned}
\mu \eta \geq \tau & \Leftrightarrow \quad \mu \eta \geq 1-\sqrt{1-\mu\left(2 \eta-\mu \kappa^{2}\right)} \\
& \Leftrightarrow \sqrt{1-\mu\left(2 \eta-\mu \kappa^{2}\right)} \geq 1-\mu \eta \\
& \Leftrightarrow 1-2 \mu \eta+\mu^{2} \kappa^{2} \geq 1-2 \mu \eta+\mu^{2} \eta^{2} \\
& \Leftrightarrow \kappa^{2} \geq \eta^{2} \\
& \Leftrightarrow \kappa \geq \eta .
\end{aligned}
$$

It is clear that

$$
\langle(\mu F-\gamma S) x-(\mu F-\gamma S) y, x-y\rangle \geq(\mu \eta-\gamma)\|x-y\|^{2}, \quad \forall x, y \in H .
$$

Hence, it follows from $0<\gamma \leq \tau \leq \mu \eta$ that $\mu F-\gamma S$ is monotone. Since $p^{*} \in \omega_{w}\left(x_{n}\right) \subset$ $\operatorname{Fix}(T) \cap \operatorname{MEP}(\Theta, \varphi) \cap \Gamma$, by Minty's lemma [1] we have

$$
\left\langle\gamma S p^{*}-\mu F p^{*}, p-p^{*}\right\rangle \leq 0, \quad \forall p \in \operatorname{Fix}(T) \cap \operatorname{MEP}(\Theta, \varphi) \cap \Gamma ;
$$

that is, $p^{*} \in \Xi$. This shows that $\omega_{w}\left(x_{n}\right) \subset \Xi$.

Theorem 3.2 Assuming the conditions in Theorem 3.1. We have:

(i) $\left\{x_{n}\right\}$ and $\left\{y_{n}\right\}$ both converge strongly to an element $x^{*} \in \operatorname{Fix}(T) \cap \operatorname{MEP}(\Theta, \varphi) \cap \Gamma$, which is a unique solution of the variational inequality

$$
\left\langle\gamma V x^{*}-\mu F x^{*}, x-x^{*}\right\rangle \leq 0, \quad \forall x \in \operatorname{Fix}(T) \cap \operatorname{MEP}(\Theta, \varphi) \cap \Gamma .
$$

(ii) $\left\{x_{n}\right\}$ and $\left\{y_{n}\right\}$ both converge strongly to a unique solution of THVI (1.6) if $\left\|x_{n}-y_{n}\right\|=o\left(\theta_{n}\right)$ in addition. 
Proof Utilizing Lemmas 2.11 and 2.12 we get from (3.15)

$$
\begin{aligned}
& \left\|x_{n+1}-p\right\|^{2} \\
& =\left\|\beta_{n} \gamma V y_{n}+(I-\mu F) T \tilde{u}_{n}-p\right\|^{2} \\
& =\left\|\beta_{n}(\gamma V p-\mu F p)+\beta_{n}\left(\gamma V y_{n}-\gamma V p\right)+\left(I-\beta_{n} \mu F\right) T \tilde{u}_{n}-\left(I-\beta_{n} \mu F\right) T p\right\|^{2} \\
& \leq\left\|\beta_{n}\left(\gamma V y_{n}-\gamma V p\right)+\left(I-\theta_{n} \mu F\right) T \tilde{u}_{n}-\left(I-\theta_{n} \mu F\right) T p\right\|^{2} \\
& +2 \beta_{n}\left\langle\gamma V p-\mu F p, x_{n+1}-p\right\rangle \\
& \leq\left[\left\|\beta_{n}\left(\gamma V y_{n}-\gamma V p\right)\right\|+\left\|\left(I-\beta_{n} \mu F\right) T \tilde{u}_{n}-\left(I-\theta_{n} \mu F\right) T p\right\|\right]^{2} \\
& +2 \beta_{n}\left\langle\gamma V p-\mu F p, x_{n+1}-p\right\rangle \\
& \leq\left[\beta_{n} \gamma \rho\left\|y_{n}-p\right\|+\left(1-\beta_{n} \tau\right)\left\|\tilde{u}_{n}-p\right\|\right]^{2}+2 \beta_{n}\left\langle\gamma V p-\mu F p, x_{n+1}-p\right\rangle \\
& \leq \beta_{n} \frac{\gamma^{2} \rho^{2}}{\tau}\left\|y_{n}-p\right\|^{2}+\left(1-\beta_{n} \tau\right)\left\|\tilde{u}_{n}-p\right\|^{2}+2 \beta_{n}\left\langle\gamma V p-\mu F p, x_{n+1}-p\right\rangle \\
& \leq \beta_{n} \frac{\gamma^{2} \rho^{2}}{\tau}\left[\left(\left\|x_{n}-p\right\|+\lambda \alpha_{n}\|p\|\right)^{2}+2 \theta_{n}\left\langle\gamma S p-\mu F p, y_{n}-p\right\rangle\right] \\
& +\left(1-\beta_{n} \tau\right)\left(\left\|x_{n}-p\right\|+\lambda \alpha_{n}\|p\|\right)^{2}+2 \beta_{n}\left\langle\gamma V p-\mu F p, x_{n+1}-p\right\rangle \\
& =\left(1-\beta_{n} \frac{\tau^{2}-\gamma^{2} \rho^{2}}{\tau}\right)\left(\left\|x_{n}-p\right\|+\lambda \alpha_{n}\|p\|\right)^{2}+2 \beta_{n} \theta_{n} \frac{\gamma^{2} \rho^{2}}{\tau}\left\langle\gamma S p-\mu F p, y_{n}-p\right\rangle \\
& +2 \beta_{n}\left\langle\gamma V p-\mu F p, x_{n+1}-p\right\rangle
\end{aligned}
$$

where $\tau=1-\sqrt{1-\mu\left(2 \eta-\mu \kappa^{2}\right)}$.

Note that $\mu F-\gamma V: H \rightarrow H$ is $(\mu \kappa+\gamma \rho)$-Lipschitzian and $(\mu \eta-\gamma \rho)$-strongly monotone, namely

$$
\|(\mu F-\gamma V) x-(\mu F-\gamma V) y\| \leq(\mu \kappa+\gamma \rho)\|x-y\|, \quad \forall x, y \in H
$$

and

$$
\langle(\mu F-\gamma V) x-(\mu F-\gamma V) y, x-y\rangle \geq(\mu \eta-\gamma \rho)\|x-y\|^{2}, \quad \forall x, y \in H .
$$

Hence there exists a unique solution $x^{*} \in \operatorname{Fix}(T) \cap \operatorname{MEP}(\Theta, \varphi) \cap \Gamma$ of the variational inequality problem

$$
\left\langle\gamma V x^{*}-\mu F x^{*}, x-x^{*}\right\rangle \leq 0, \quad \forall x \in \operatorname{Fix}(T) \cap \operatorname{MEP}(\Theta, \varphi) \cap \Gamma .
$$

Since the sequence $\left\{x_{n}\right\}$ is bounded, there exists a subsequence $\left\{x_{n_{i}}\right\}$ of $\left\{x_{n}\right\}$ such that

$$
\limsup _{n \rightarrow \infty}\left\langle\gamma V x^{*}-\mu F x^{*}, x_{n}-x^{*}\right\rangle=\lim _{i \rightarrow \infty}\left\langle\gamma V x^{*}-\mu F x^{*}, x_{n_{i}}-x^{*}\right\rangle .
$$

Also, since $H$ is reflexive and $\left\{x_{n}\right\}$ is bounded, without loss of generality we may assume that $x_{n_{i}} \rightarrow \bar{x} \in \operatorname{Fix}(T) \cap \operatorname{MEP}(\Theta, \varphi) \cap \Gamma$ (due to Theorem 3.1(i)). Taking into consideration 
that $x^{*}$ is the unique solution of VIP (3.17), we obtain from (3.18)

$$
\begin{aligned}
& \limsup _{n \rightarrow \infty}\left\langle\gamma V x^{*}-\mu F x^{*}, x_{n+1}-x^{*}\right\rangle \\
& \quad=\limsup _{n \rightarrow \infty}\left(\left\langle\gamma V x^{*}-\mu F x^{*}, x_{n}-x^{*}\right\rangle+\left\langle\gamma V x^{*}-\mu F x^{*}, x_{n+1}-x_{n}\right\rangle\right) \\
& =\limsup _{n \rightarrow \infty}\left\langle\gamma V x^{*}-\mu F x^{*}, x_{n}-x^{*}\right\rangle=\lim _{i \rightarrow \infty}\left\langle\gamma V x^{*}-\mu F x^{*}, x_{n_{i}}-x^{*}\right\rangle \\
& =\left\langle\gamma V x^{*}-\mu F x^{*}, \bar{x}-x^{*}\right\rangle \leq 0 .
\end{aligned}
$$

Putting $p=x^{*}$, from (3.16) we conclude that

$$
\begin{aligned}
\left\|x_{n+1}-x^{*}\right\|^{2} & \\
\leq & \left(1-\beta_{n} \frac{\tau^{2}-\gamma^{2} \rho^{2}}{\tau}\right)\left(\left\|x_{n}-x^{*}\right\|+\lambda \alpha_{n}\left\|x^{*}\right\|\right)^{2} \\
& +2 \beta_{n} \theta_{n} \frac{\gamma^{2} \rho^{2}}{\tau}\left\|\gamma S x^{*}-\mu F x^{*}\right\|\left\|y_{n}-x^{*}\right\|+2 \beta_{n}\left\langle\gamma V x^{*}-\mu F x^{*}, x_{n+1}-x^{*}\right\rangle \\
\leq & \left(1-\beta_{n} \frac{\tau^{2}-\gamma^{2} \rho^{2}}{\tau}\right)\left\|x_{n}-x^{*}\right\|^{2}+\lambda \alpha_{n}\left\|x^{*}\right\|\left(2\left\|x_{n}-x^{*}\right\|+\lambda \alpha_{n}\left\|x^{*}\right\|\right) \\
& +2 \beta_{n} \theta_{n} \frac{\gamma^{2} \rho^{2}}{\tau}\left\|\gamma S x^{*}-\mu F x^{*}\right\|\left\|y_{n}-x^{*}\right\|+2 \beta_{n}\left\langle\gamma V x^{*}-\mu F x^{*}, x_{n+1}-x^{*}\right\rangle \\
= & \left(1-\beta_{n} \frac{\tau^{2}-\gamma^{2} \rho^{2}}{\tau}\right)\left\|x_{n}-x^{*}\right\|^{2}+\beta_{n} \frac{\tau^{2}-\gamma^{2} \rho^{2}}{\tau} \\
& +\frac{\tau}{\tau^{2}-\gamma^{2} \rho^{2}}\left\{2 \theta_{n} \frac{\gamma^{2} \rho^{2}}{\tau}\left\|\gamma S x^{*}-\mu F x^{*}\right\|\left\|y_{n}-x^{*}\right\|\right. \\
& \left.+2\left\langle\gamma V x^{*}-\mu F x^{*}, x_{n+1}-x^{*}\right\rangle\right\}+\alpha_{n} \lambda\left\|x^{*}\right\|\left(2\left\|x_{n}-x^{*}\right\|+\lambda \alpha_{n}\left\|x^{*}\right\|\right) .
\end{aligned}
$$

Since $\sum_{n=0}^{\infty} \beta_{n}=+\infty, \sum_{n=0}^{\infty} \alpha_{n}<+\infty$, and $\theta_{n} \rightarrow 0$ as $n \rightarrow \infty$, it follows from (3.19) that $\sum_{n=0}^{\infty} \beta_{n} \frac{\tau^{2}-\gamma^{2} \rho^{2}}{\tau}=+\infty, \sum_{n=0}^{\infty} \alpha_{n} \lambda\left\|x^{*}\right\|\left(2\left\|x_{n}-x^{*}\right\|+\lambda \alpha_{n}\left\|x^{*}\right\|\right)<+\infty$, and

$$
\limsup _{n \rightarrow \infty} \frac{\tau}{\tau^{2}-\gamma^{2} \rho^{2}}\left\{2 \theta_{n} \frac{\gamma^{2} \rho^{2}}{\tau}\left\|\gamma S x^{*}-\mu F x^{*}\right\|\left\|y_{n}-x^{*}\right\|+2\left\langle\gamma V x^{*}-\mu F x^{*}, x_{n+1}-x^{*}\right\rangle\right\} \leq 0 .
$$

Applying Lemma 2.7 to (3.20), we get

$$
\lim _{n \rightarrow \infty}\left\|x_{n}-x^{*}\right\|=0
$$

This, together with $\left\|x_{n}-y_{n}\right\| \rightarrow 0$, implies that

$$
\lim _{n \rightarrow \infty}\left\|y_{n}-x^{*}\right\|=0
$$

From now on, we suppose that $\left\|x_{n}-y_{n}\right\|=o\left(\theta_{n}\right)$. Then by Theorem 3.1(ii) we know that $\omega_{w}\left(x_{n}\right) \subset \Xi$. Since $\mu F-\gamma V: H \rightarrow H$ is $(\mu \kappa+\gamma \rho)$-Lipschitzian and $(\mu \eta-\gamma \rho)$-strongly monotone, there exists a unique solution $x^{*} \in \Xi$ of the variational inequality problem

$$
\left\langle\gamma V x^{*}-\mu F x^{*}, x-x^{*}\right\rangle \leq 0, \quad \forall x \in \Xi .
$$


Since the sequence $\left\{x_{n}\right\}$ is bounded, there exists a subsequence $\left\{x_{n_{i}}\right\}$ of $\left\{x_{n}\right\}$ such that

$$
\limsup _{n \rightarrow \infty}\left\langle\gamma V x^{*}-\mu F x^{*}, x_{n}-x^{*}\right\rangle=\lim _{i \rightarrow \infty}\left\langle\gamma V x^{*}-\mu F x^{*}, x_{n_{i}}-x^{*}\right\rangle .
$$

Again, since $H$ is reflexive and $\left\{x_{n}\right\}$ is bounded, without loss of generality we may assume that $x_{n_{i}} \rightarrow \bar{x} \in \Xi$ (due to Theorem 3.1(ii)). Taking into account that $x^{*}$ is the unique solution of VIP (3.21), we deduce from (3.22) that

$$
\limsup _{n \rightarrow \infty}\left\langle\gamma V x^{*}-\mu F x^{*}, x_{n+1}-x^{*}\right\rangle \leq\left\langle\gamma V x^{*}-\mu F x^{*}, \bar{x}-x^{*}\right\rangle \leq 0 .
$$

Putting $p=x^{*}$, from (3.16) we immediately infer that

$$
\begin{aligned}
& \left\|x_{n+1}-x^{*}\right\|^{2} \\
& \leq\left(1-\beta_{n} \frac{\tau^{2}-\gamma^{2} \rho^{2}}{\tau}\right)\left\|x_{n}-x^{*}\right\|^{2}+\beta_{n} \frac{\tau^{2}-\gamma^{2} \rho^{2}}{\tau} \\
& \quad \cdot \frac{\tau}{\tau^{2}-\gamma^{2} \rho^{2}}\left\{2 \theta_{n} \frac{\gamma^{2} \rho^{2}}{\tau}\left\|\gamma S x^{*}-\mu F x^{*}\right\|\left\|y_{n}-x^{*}\right\|\right. \\
& \left.\quad+2\left\langle\gamma V x^{*}-\mu F x^{*}, x_{n+1}-x^{*}\right\rangle\right\}+\alpha_{n} \lambda\left\|x^{*}\right\|\left(2\left\|x_{n}-x^{*}\right\|+\lambda \alpha_{n}\left\|x^{*}\right\|\right) .
\end{aligned}
$$

Repeating the same arguments as above, we can readily see that

$$
\lim _{n \rightarrow \infty}\left\|x_{n}-x^{*}\right\|=0
$$

which, together with $\left\|x_{n}-y_{n}\right\| \rightarrow 0$, yields

$$
\lim _{n \rightarrow \infty}\left\|y_{n}-x^{*}\right\|=0
$$

This completes the proof.

Remark 3.3 Our iterative algorithm (3.1) is very different from Xu's iterative ones in [2], and Yao et al.'s iterative one in [8]. Here, the two-step iterative scheme in [8] for two nonexpansive mappings and the gradient-projection iterative schemes in [2] for MP (1.1) are extended to develop our three-step iterative scheme (3.1) with regularization for the THVI (1.6). It is worth pointing out that without assuming the conditions that $\left\|x_{n}-y_{n}\right\|=o\left(\theta_{n}\right)$ and that $\|x-T x\| \geq k \operatorname{Dist}(x, \operatorname{Fix}(T)), \forall x \in C$ for some constant $k>0$, our three-step iterative scheme (3.1) converges strongly to an element $x^{*} \in \operatorname{Fix}(T) \cap \operatorname{MEP}(\Theta, \varphi) \cap \Gamma$, which is a unique solution of the variational inequality

$$
\left\langle\gamma V x^{*}-\mu F x^{*}, x-x^{*}\right\rangle \leq 0, \quad \forall x \in \operatorname{Fix}(T) \cap \operatorname{MEP}(\Theta, \varphi) \cap \Gamma .
$$

See Theorem 3.2(i).

Remark 3.4 As an example, we consider the following sequences:

(a) $\alpha_{n}=\frac{1}{n^{1+s+t}}, \beta_{n}=\frac{1}{n^{s}}$, and $\theta_{n}=\frac{1}{n^{t}}$ where $t \in\left(0, \frac{1}{3}\right]$ and $s \in(t, 2 t)$ or $t \in\left(\frac{1}{3}, \frac{1}{2}\right)$, $s \in(t, 1-t)$; 
(b) $r_{n}=\frac{1}{2}+\frac{1}{n^{1+s+t}}$.

They satisfy the hypotheses on the parameter sequences in Theorems 3.1 and 3.2.

Remark 3.5 Our Theorems 3.1 and 3.2 improve, extend, supplement, and develop [8, Theorems 3.1 and 3.2] and [2, Theorems 5.2 and 6.1] in the following aspects:

(a) Our THVI (1.6) with the unique solution $x^{*} \in \Xi$ satisfying

$$
x^{*}=P_{\operatorname{Fix}(T) \cap \operatorname{Fix}(\Gamma) \cap \Xi}(I-(\mu F-\gamma S)) x^{*}
$$

is more general than the problem of finding an element $\tilde{x} \in C$ satisfying $\tilde{x}=P_{\mathrm{Fix}(T)} S \tilde{x}$ in [8] and the problem of finding an element $\tilde{x} \in \boldsymbol{\Xi}$ in [2].

(b) Our three-step iterative algorithm (3.1) for THVI (1.6) is more flexible, more advantageous and more subtle than Xu's iterative ones in [2] and than Yao et al.'s two-step iterative one in [8], because, e.g., it drops the requirement of $\|x-T x\| \geq k \operatorname{Dist}(x, \operatorname{Fix}(T)), \forall x \in C$ for some $k>0$ in [8, Theorem 3.2(v)].

(c) The arguments and techniques in our Theorems 3.1 and 3.2 are very different from the ones in [8, Theorems 3.1 and 3.2] and in [2, Theorems 5.2 and 6.1] because we utilize the properties of resolvent operators and maximal monotone mappings (Lemmas 2.5, 2.6 and 2.13), the convergence criteria of real sequences (Lemma 2.7), and the contractive coefficient estimates for the contractions associated with nonexpansive mappings (Lemma 2.12).

(d) Compared with the proofs of [2, Theorems 5.2 and 6.1], the proofs of our Theorems 3.1 and 3.2 derive $\lim _{n \rightarrow \infty}\left\|u_{n}-P_{C}\left(I-\lambda \nabla f_{\alpha_{n}}\right) u_{n}\right\|=0$ via the argument showing $\lim _{n \rightarrow \infty}\left\|\nabla f_{\alpha_{n}}\left(u_{n}\right)-\nabla f(p)\right\|=0, \forall p \in \operatorname{Fix}(T) \cap \operatorname{MEP}(\Theta, \varphi) \cap \Gamma$ (see Step 3 in the proof of Theorem 3.1).

\section{Competing interests}

The authors declare that they have no competing interests.

\section{Authors' contributions}

All authors contributed equally and significantly in writing this paper. All authors read and approved the final manuscript.

\section{Author details}

'Department of Mathematics, Shanghai Normal University, Shanghai, 200234, China. ${ }^{2}$ Scientific Computing Key Laboratory of Shanghai Universities, Shanghai, 200234, China. ${ }^{3}$ Department of Applied Mathematics, National Sun Yat-sen University, Kaohsiung, 804, Taiwan. ${ }^{4}$ Center for General Education, Kaohsiung Medical University, Kaohsiung, 807, Taiwan. ${ }^{5}$ Center for Fundamental Science, Kaohsiung Medical University, Kaohsiung, 807, Taiwan. ${ }^{6}$ Department of Mathematics, King Abdulaziz University, P.O. Box 80203, Jeddah, 21589, Saudi Arabia.

\section{Acknowledgements}

Lu-Chuan Ceng is partially supported by the National Science Foundation of China (11071169), Innovation Program of Shanghai Municipal Education Commission (09ZZ133) and Leading Academic Discipline Project of Shanghai Normal University (DZL707). Ngai-Ching Wong is partially supported by the Taiwan MOST grant 102-2115-M-110-002-MY2. Jen-Chih Yao is partially supported by the Taiwan MOST grant 102-2111-E-037-004-MY3. Both Ngai-Ching Wong and Jen-Chih Yao are also partially supported by the NSYSU-KMU joint venture 103-P013.

Received: 31 August 2014 Accepted: 24 November 2014 Published: 12 Dec 2014

\section{References}

1. Goebel, K, Kirk, WA: Topics on Metric Fixed Point Theory. Cambridge Studies in Advanced Mathematics, vol. 28. Cambridge University Press, Cambridge (1990)

2. Xu, H-K: Averaged mappings and the gradient-projection algorithm. J. Optim. Theory Appl. 150, 360-378 (2011)

3. $\mathrm{Xu}, \mathrm{H}-\mathrm{K}$ : Iterative methods for the split feasibility problem in infinite-dimensional Hilbert spaces. Inverse Problems 26 , 105018 (2010)

4. Ceng, L-C, Wang, C-Y, Yao, J-C: Strong convergence theorems by a relaxed extragradient method for a general system of variational inequalities. Math. Methods Oper. Res. 67, 375-390 (2008) 
5. Ceng, L-C, Yao, J-C: An extragradient-like approximation method for variational inequality problems and fixed point problems. Appl. Math. Comput. 190, 205-215 (2007)

6. Glowinski, R: Numerical Methods for Nonlinear Variational Problems. Springer, New York (1984)

7. Kinderlehrer, D, Stampacchia, G: An Introduction to Variational Inequalities and Their Applications. Academic Press, New York (1980)

8. Yao, Y, Liou, Y-C, Marino, G: Two-step iterative algorithms for hierarchical fixed point problems and variational inequality problems. J. Appl. Math. Comput. 31(1-2), 433-445 (2009)

9. Moudafi, A, Maingé, P-E: Towards viscosity approximations of hierarchical fixed points problems. Fixed Point Theory Appl. 2006, Article ID 95453 (2006)

10. Moudafi, A, Maingé, P-E: Strong convergence of an iterative method for hierarchical fixed point problems. Pac. J. Optim. 3(3), 529-538 (2007)

11. Moudafi, A: Viscosity approximation methods for fixed-points problems. J. Math. Anal. Appl. 241(1), 46-55 (2000)

12. Xu, H-K: Viscosity approximation methods for nonexpansive mappings. J. Math. Anal. Appl. 298, 279-291 (2004)

13. Ceng, L-C, Yao, J-C: A hybrid iterative scheme for mixed equilibrium problems and fixed point problems. J. Comput. Appl. Math. 214, 186-201 (2008)

14. Blum, E, Oettli, W: From optimization and variational inequalities to equilibrium problems. Math. Stud. 63, 123-145 (1994)

15. Peng, J-W, Yao, J-C: A new hybrid-extragradient method for generalized mixed equilibrium problems, fixed point problems and variational inequality problems. Taiwan. J. Math. 12,1401-1432 (2008)

16. liduka, $\mathrm{H}$ : Strong convergence for an iterative method for the triple-hierarchical constrained optimization problem Nonlinear Anal. 71, 1292-1297 (2010)

17. liduka, H: Iterative algorithm for solving triple-hierarchical constrained optimization problem. J. Optim. Theory Appl. 148, 580-592 (2011)

18. Ceng, L-C, Ansari, QH, Yao, J-C: Iterative methods for triple hierarchical variational inequalities in Hilbert spaces. J. Optim. Theory Appl. 151, 489-512 (2011)

19. Ansari, QH, Ceng, L-C, Gupta, H: Triple hierarchical variational inequalities. In: Ansari, QH (ed.) Nonlinear Analysis: Approximation Theory, Optimization and Applications, pp. 231-280. Birkhäuser, Basel (2014)

20. Ceng, L-C, Ansari, QH, Wen, C-F: Hybrid steepest-descent viscosity method for triple hierarchical variational inequalities. Abstr. Appl. Anal. 2012, Article ID 907105 (2012)

21. Ceng, L-C, Ansari, QH, Yao, J-C: Relaxed hybrid steepest-descent methods with variable parameters for triple-hierarchical variational inequalities. Appl. Anal. 91(10), 1793-1810 (2012)

22. Kong, Z-R, Ceng, L-C, Pang, CT, Ansari, QH: Multi-step hybrid extragradient method for triple hierarchical variational inequalities. Abstr. Appl. Anal. 2013, Article ID 718624 (2013)

23. Byrne, C: A unified treatment of some iterative algorithms in signal processing and image reconstruction. Inverse Problems 20, 103-120 (2004)

24. Combettes, PL: Solving monotone inclusions via compositions of nonexpansive averaged operators. Optimization 53(5-6), 475-504 (2004)

25. Xu, H-K: Iterative algorithms for nonlinear operators. J. Lond. Math. Soc. 66(2), 240-256 (2002)

26. Reineermann, J: Über fixpunkte kontrahierender abbildungen und schwach konvergente Toeplitz-verfahren. Arch. Math. (Basel) 20, 59-64 (1969)

27. $\mathrm{Xu}, \mathrm{H}-\mathrm{K}, \mathrm{Kim}, \mathrm{T}-\mathrm{H}$ : Convergence of hybrid steepest-descent methods for variational inequalities. J. Optim. Theory Appl. 119, 185-201 (2003)

28. Rockafellar, RT: On the maximality of sums of nonlinear monotone operators. Trans. Am. Math. Soc. 149, 75-88 (1970)

10.1186/1029-242X-2014-490

Cite this article as: Ceng et al.: Regularized hybrid iterative algorithms for triple hierarchical variational inequalities. Journal of Inequalities and Applications 2014, 2014:490

\section{Submit your manuscript to a SpringerOpen ${ }^{\circ}$ journal and benefit from:}

- Convenient online submission

- Rigorous peer review

- Immediate publication on acceptance

- Open access: articles freely available online

- High visibility within the field

- Retaining the copyright to your article 